\title{
A RELAXATION RESULT IN BV FOR INTEGRAL FUNCTIONALS WITH DISCONTINUOUS INTEGRANDS
}

\author{
Micol Amar ${ }^{1}$, Virginia De CicCo $^{1}$ and Nicola Fusco ${ }^{2}$
}

\begin{abstract}
We prove a relaxation theorem in BV for a non coercive functional with linear growth. No continuity of the integrand with respect to the spatial variable is assumed.
\end{abstract}

Mathematics Subject Classification. 49J45, 26B30.

Received November 11, 2005. Revised March 28, 2006.

\section{INTRODUCTION}

In this paper we study the relaxation in $\mathrm{BV}(\Omega)$ of an integral functional of the type

$$
F(u)=\int_{\Omega} f(x, u(x), \nabla u(x)) \mathrm{d} x
$$

where $u$ is a scalar function from $W^{1,1}(\Omega)$.

In recent years there has been a renewed interest in the $L^{1}$-lower semicontinuity of such an integral functional and of its BV counterpart

$$
\mathcal{F}(u)=\int_{\Omega} f(x, u, \nabla u) \mathrm{d} x+\int_{\Omega} f^{\infty}\left(x, \widetilde{u}, \frac{D^{c} u}{\left|D^{c} u\right|}\right) d\left|D^{c} u\right|+\int_{J_{u} \cap \Omega}\left(\int_{u^{-}(x)}^{u^{+}(x)} f^{\infty}\left(x, s, \nu_{u}\right) \mathrm{d} s\right) \mathrm{d} \mathcal{H}^{N-1}(x)
$$

with the aim of weakening the regularity assumptions on the integrand $f$ with respect to the spatial variable $x$ (see $[7-9,18,20,21])$. Roughly speaking, one can show that the $L^{1}$-lower semicontinuity still holds if one replaces the classical continuity and coerciveness assumptions with the weak differentiability of $f$ with respect to $x$. Therefore the results proved in the above papers suggest that a similar assumption should be also enough to prove that the relaxation in $\mathrm{BV}$ of the functional $F$ is represented by $\mathcal{F}$.

In this paper we prove that this representation formula actually holds (see Th. 6.1) under the assumption that for all $(s, \xi) \in \mathbb{R} \times \mathbb{R}^{N}$ the function $f(\cdot, s, \xi)$ is weakly differentiable and coincides $\mathcal{H}^{N-1}$-a.e. with its precise representative, i.e., it is $\mathcal{H}^{N-1}$-a.e. approximately continuous in $\Omega$. Notice that though the functional $F$ does not change its values if we modify $f$ in a subset of zero Lebesgue measure of $\Omega$, this modification may affect the values of $\mathcal{F}$ on $\operatorname{BV}(\Omega)$. Therefore, if $f$ is not assumed to be $\mathcal{H}^{N-1}$-a.e. approximately continuous with respect to $x$, then it is not true in general that the relaxation of $F$ is represented by $\mathcal{F}$. On the other hand the approximate continuity alone is not enough to assure the relaxation result, as shown in a counterexample given in [1]. In that

\footnotetext{
Keywords and phrases. Lower semicontinuity, relaxation, BV-functions, blow-up.

1 Dipartimento di Metodi e Modelli Matematici, via A. Scarpa 16, 00161 Roma, Italy; amar@dmmm.uniroma1.it; decicco@dmmm.uniroma1.it

2 Dipartimento di Matematica e Applicazioni Monte Sant'Angelo, via Cintia, 80126 Napoli, Italy; n.fusco@unina.it
}

(C) EDP Sciences, SMAI 2007 
paper the representation formula proved here has been obtained in the particular case where the integrand admits a separate dependence on the spatial and the gradient variables. In that case the authors prove the relaxation result by suitably refining the techniques introduced in [5] through the use of a new Reshetnyak-type theorem which applies only to measures which are gradients of BV-function, but does not require the continuity of the integrand. Unfortunately, this technique does not work for a general integrand like the ones considered here.

Thus, we have to follow another approach to relaxation by using the blow-up technique introduced by Fonseca and Müller in [16] and [17] (see also [3]). However, in all these papers the use of this technique relies strongly on the continuity of the integrand with respect to $x$, an assumption that here is replaced by the $\mathcal{H}^{N-1}$-a.e. approximate continuity and weak differentiability in $x$.

This fact introduces some relevant difficulties and requires a delicate study of the approximate continuity of $(N-1)$-dimensional restrictions of BV-functions. Differently from the usual continuity, the approximate continuity is not inherited by the sections of measurable functions. However, in the first part of this paper we prove that given a $\mathcal{H}^{N-1}$-almost everywhere approximately continuous BV-function, its sections keep the same property, as long as we restrict them to a countably $\mathcal{H}^{N-1}$-rectifiable set whose normal is "never" orthogonal to the hyperplane with respect to whom the sections are taken (see Th. 4.6). This theorem is the main tool needed for dealing with the jump part of the functional via the blow-up technique. More precisely, given a jump point $x_{0}$ of a BV-function $u$, we study the behaviour of the integrand on the tangent hyperplane $\Pi$ to the jump set at $x_{0}$. If the restriction of the integrand to $\Pi$ is not approximately continuous, we have to approximate $\Pi$ with a sequence of "good" hyperplanes (where the restriction of $f$ is approximately continuous). In fact, in Proposition 6.5 we prove that this property holds at $\mathcal{H}^{N-1}$-point $x_{0}$ of the jump set of $u$.

The paper is organized as follows: Section 2 is devoted to notations; in Section 3 we recall some properties of $\mathrm{BV}$-functions and some results of geometric measure theory needed for the sequel. In Section 4 we carry on a thorough analysis of the fine properties of the $(N-1)$-dimensional sections of BV-functions. In Section 5 we set the problem and state some technical lemmas; moreover, we discuss some properties of the recession function that do not follow from the corresponding ones of integrand (see Ex. 5.3). Finally, in Section 6 we state and prove our main result, i.e., the relaxation theorem.

\section{Notation}

Throughout the paper, $N \geq 2$ is a fixed integer and the letter $c$ denotes a strictly positive constant, whose value may vary from line to line. Given $x_{0} \in \mathbb{R}^{N}$ and $\rho>0, B_{\rho}\left(x_{0}\right)$ denotes the ball in $\mathbb{R}^{N}$ centered in $x_{0}$ with radius $\rho$, while $\mathbb{S}^{N-1}$ is the unit sphere of $\mathbb{R}^{N}$.

Let $\Omega$ be a bounded open set in $\mathbb{R}^{N}$. We denote by $\mathcal{A}(\Omega)$ the family of all bounded open subsets $A$ of $\Omega$ and by $\mathcal{B}(\Omega)$ the $\sigma$-algebra of all Borel subsets $B$ of $\Omega$. Moreover, $\mathcal{M}\left(\Omega ; \mathbb{R}^{N}\right)$ is the space of the $\mathbb{R}^{N}$-valued $\operatorname{Radon}$ measures on $\Omega$; in particular, $\mathcal{M}(\Omega):=\mathcal{M}(\Omega ; \mathbb{R})$.

As usual, $\mathcal{L}^{N}$ stands for the outer Lebesgue measure on $\mathbb{R}^{N}$ and $\mathcal{H}^{k}$ for the $k$-dimensional Hausdorff measure on $\mathbb{R}^{N}$. The Lebesgue measure of the unit ball in $\mathbb{R}^{N}$ is denoted by $\omega_{N}$, hence $\mathcal{L}^{N}\left(B_{\rho}\left(x_{0}\right)\right)=\omega_{N} \rho^{N}$.

Given a direction $\nu \in \mathbb{S}^{N-1}$, every point $x \in \mathbb{R}^{N}$ can be decomposed as $x=\left(x_{\nu}^{\perp}, x_{\nu}\right)$, with $x_{\nu}=\langle x, \nu\rangle \nu$ and $x_{\nu}^{\perp}=x-x_{\nu}$. By $\pi_{\nu^{\perp}}$ we denote the projection of $\mathbb{R}^{N}$ onto the plane through the origin orthogonal to $\nu$ and $\pi_{\nu}$ denotes the projection of $\mathbb{R}^{N}$ over the line through the origin in the direction $\nu$. We shall often identify $\pi_{\nu^{\perp}}\left(\mathbb{R}^{N}\right)$ with $\mathbb{R}^{N-1}$ and $\pi_{\nu}\left(\mathbb{R}^{N}\right)$ with $\mathbb{R}$, so that, for instance, the $\mathcal{H}^{N-1}$-measure on $\pi_{\nu^{\perp}}\left(\mathbb{R}^{N}\right)$ will be identified with $\mathcal{L}^{N-1}$. Finally, if $E$ is a given subset of $\mathbb{R}^{N}$, we set

$$
E_{x_{\nu}^{\perp}}=\left\{x_{\nu} \in \pi_{\nu}(E):\left(x_{\nu}^{\perp}, x_{\nu}\right) \in E\right\} \quad \text { and } \quad E_{x_{\nu}}=\left\{x_{\nu}^{\perp} \in \pi_{\nu^{\perp}}(E):\left(x_{\nu}^{\perp}, x_{\nu}\right) \in E\right\} .
$$

Similarly, if $g: \mathbb{R}^{N} \rightarrow \mathbb{R}$ is a given function, for every $x_{\nu}^{\perp} \in \mathbb{R}^{N-1}$, we denote by $g_{x_{\nu}^{\perp}}$ the restriction of the function $g$ to $\mathbb{R}$; i.e., the function $x_{\nu} \in \mathbb{R} \mapsto g\left(x_{\nu}^{\perp}, x_{\nu}\right)$; for every $x_{\nu} \in \mathbb{R}$, the restriction $g_{x_{\nu}}$ is defined analogously.

When $\nu=\mathrm{e}_{N}$, we simply write $\pi_{N-1}, \pi_{1},\left(x^{\prime}, y\right), E_{x^{\prime}}, E_{y}, g_{x^{\prime}}, g_{y}$, instead of $\pi_{\mathrm{e}_{N}^{\perp}}, \pi_{\mathrm{e}_{N}},\left(x_{\mathrm{e}_{N}}^{\perp}, x_{\mathrm{e}_{N}}\right), E_{x_{\mathrm{e}_{N}}^{\perp}}, E_{x_{\mathrm{e}_{N}}}$, $g_{x_{\mathrm{e}_{N}}^{\perp}}, g_{x_{\mathrm{e}_{N}}}$. 


\section{BASIC PROPERTIES OF BV-FUNCTIONS AND A COAREA FORMULA}

Let $u \in L_{\text {loc }}^{1}(\Omega)$; we say that $u$ has an approximate limit at $x \in \Omega$ if there exists $z \in \mathbb{R}$ such that

$$
\lim _{\varepsilon \rightarrow 0^{+}} f_{B_{\varepsilon}(x)}|u(y)-z| \mathrm{d} x=0
$$

where $f_{B_{\varepsilon}(x)}$ stands for $\frac{1}{\mathcal{L}^{N}\left(B_{\varepsilon}(x)\right)} \int_{B_{\varepsilon}(x)}$. Let $S_{u}$ be the set of points where the previous property does not hold, the so-called approximate discontinuity set. Note that it is a Borel set. If $x \notin S_{u}, z$ is uniquely determined, it is called the approximate limit of $u$ at $x$ and it is denoted by $\widetilde{u}(x)$. We recall that $\widetilde{u}: \Omega \backslash S_{u} \rightarrow \mathbb{R}$ is a Borel function.

We say that $u$ is approximately continuous at $x$ if $x \notin S_{u}$ and $u(x)=\widetilde{u}(x)$. Clearly, $x$ is a point of approximate continuity of $u$ if and only if is a Lebesgue point of $u$ and since $\mathcal{L}^{N}$-almost every $x \in \Omega$ is a Lebesgue point, $\mathcal{L}^{N}\left(S_{u}\right)=0$. Notice that in general the above definition of approximate continuity is stronger than the usual one given by Federer (see [13]). However, if $u \in L_{\mathrm{loc}}^{\infty}(\Omega)$ the two notions agree.

We say that $x_{0} \in S_{u}$ is an approximate jump point of $u$ if there exist $a, b \in \mathbb{R}$ and $\nu \in \mathbb{S}^{N-1}$, such that $a \neq b$ and

$$
\lim _{\varepsilon \rightarrow 0^{+}} f_{B_{\varepsilon}^{+}\left(x_{0}, \nu\right)}|u(x)-a| \mathrm{d} x=0, \quad \lim _{\varepsilon \rightarrow 0^{+}} f_{B_{\varepsilon}^{-}\left(x_{0}, \nu\right)}|u(x)-b| \mathrm{d} x=0,
$$

where $B_{\varepsilon}^{ \pm}\left(x_{0}, \nu\right)=x_{0}+\varepsilon B_{\nu}^{ \pm}$and $B_{\nu}^{ \pm}=\left\{x \in B_{1}(0):\langle x, \nu\rangle \gtrless 0\right\}$. The triplet $(a, b, \nu)$, uniquely determined by the previous definition up to a permutation of $a, b$ and a change of sign of $\nu$, is denoted by $\left(u^{+}\left(x_{0}\right), u^{-}\left(x_{0}\right), \nu_{u}\left(x_{0}\right)\right)$. We adopt the convention that $u^{+}\left(x_{0}\right)>u^{-}\left(x_{0}\right)$. The set of approximate jump points is denoted by $J_{u}$ and is a Borel set. The quantity $u^{+}-u^{-}$is the jump of $u$ across the interface $J_{u}$ and $\nu_{u}$ is the direction of the jump.

The space $\operatorname{BV}(\Omega)$ is defined as the space of all functions $u: \Omega \rightarrow \mathbb{R}$ belonging to $L^{1}(\Omega)$ whose distributional gradient $D u$ is an $\mathbb{R}^{N}$-valued Radon measure (i.e., $D u \in \mathcal{M}\left(\Omega ; \mathbb{R}^{N}\right)$ ) with total variation $|D u|$ bounded in $\Omega$. We indicate by $D^{a} u$ and $D^{s} u$ the absolutely continuous and the singular part of the measure $D u$ with respect to the Lebesgue measure. We recall that $D^{a} u$ and $D^{s} u$ are mutually singular, moreover we can write

$$
D u=D^{a} u+D^{s} u \quad \text { and } \quad D^{a} u=\nabla u \mathcal{L}^{N}
$$

where $\nabla u$ is the Radon-Nikodým derivative of $D^{a} u$ with respect to the Lebesgue measure. In particular,

$$
D^{s} u=D^{c} u+\left(u^{+}-u^{-}\right) \nu_{u} \mathcal{H}^{N-1}\left\lfloor J_{u}\right.
$$

and $J_{u}$ is a countably $\mathcal{H}^{N-1}$-rectifiable Borel set (see [2], Def. 2.57) contained in $S_{u}$, such that $\mathcal{H}^{N-1}\left(S_{u} \backslash J_{u}\right)=0$. The remaining part $D^{c} u$ is called the Cantor part of $D u$.

Remark 3.1. Since, for every $u \in \operatorname{BV}(\Omega), J_{u}$ is a countably $\mathcal{H}^{N-1}$-rectifiable set, hence $\sigma$-finite with respect to $\mathcal{H}^{N-1}$, it follows that the set

$$
\left\{\nu \in \mathbb{S}^{N-1}: \mathcal{H}^{N-1}\left(\left\{x \in J_{u}: \nu_{u}(x)= \pm \nu\right\}\right)>0\right\}
$$

is at most countable.

Finally, we define the precise representative of a function $u \in \in L_{\mathrm{loc}}^{1}(\Omega)$ as

$$
u^{*}(x)= \begin{cases}\widetilde{u}(x) & \text { if } x \in \Omega \backslash S_{u} \\ \frac{u^{+}(x)+u^{-}(x)}{2} & \text { if } x \in J_{u} \\ 0 & \text { if } x \in S_{u} \backslash J_{u}\end{cases}
$$

Clearly, $u^{*}: \Omega \rightarrow \mathbb{R}$ is a Borel function coinciding $\mathcal{L}^{N}$-almost everywhere with $\widetilde{u}$ and $u$.

Let us recall that if $S \subset \mathbb{R}^{N}$ is a countably $\mathcal{H}^{N-1}$-rectifiable set, then for $\mathcal{H}^{N-1}$-a.e. $x \in S$ there exists the approximate tangent plane $\pi_{x}^{S}$ to $S$ at $x$ (see [2], Th. 2.83). A unit vector orthogonal to $\pi_{x}^{S}$ is called an approximate 
normal to $S$ at $x$ and denoted by $\nu^{S}(x)$. Notice that if $S$ is the jump set $J_{u}$ of some BV function $u$, then ([2], Th. 3.59) $\nu^{J_{u}}(x)= \pm \nu_{u}(x)$ for $\mathcal{H}^{N-1}$-a.e. $x \in J_{u}$.

Let $1 \leq k \leq N-1$ be a given integer. By $\pi_{k, N}: \mathbb{R}^{N} \rightarrow \mathbb{R}^{k}$ we denote the projection of $\mathbb{R}^{N}$ over the first $k$ components. The formula (3.1) below is a consequence of the general coarea formula for rectifiable set [2], Theorem 2.93, and will be used in the sequel. For the reader's convenience we give an explicit proof.

Theorem 3.2. Let $S$ be a countably $\mathcal{H}^{N-1}$-rectifiable subset of $\mathbb{R}^{N}$ and $g: \mathbb{R}^{N} \rightarrow[0,+\infty]$ a Borel function. Then, for any integer $1 \leq k \leq N-1$,

$$
\int_{S} g(x) \sqrt{\sum_{i=k+1}^{N}\left|\left\langle\nu^{S}(x), e_{i}\right\rangle\right|^{2}} \mathrm{~d} \mathcal{H}^{N-1}(x)=\int_{\mathbb{R}^{k}} \mathrm{~d} t \int_{\pi_{k, N}^{-1}(t) \cap S} g(x) \mathrm{d} \mathcal{H}^{N-k-1}(x) .
$$

Proof. From the coarea formula for rectifiable sets [2], Theorem 2.93 we have that

$$
\int_{S} g(x) C_{k} L_{x} \mathrm{~d} \mathcal{H}^{N-1}(x)=\int_{\mathbb{R}^{k}} \mathrm{~d} t \int_{\pi_{k, N}^{-1}(t) \cap S} g(x) \mathrm{d} \mathcal{H}^{N-k-1}(x),
$$

where

$$
C_{k} L_{x}:=\sqrt{\operatorname{det}\left(L_{x} \circ L_{x}^{*}\right)}
$$

$L_{x}: \pi_{x}^{S} \rightarrow \mathbb{R}^{k}$ is the differential of $\pi_{k, N}$ on $S$ at $x$ and $L_{x}^{*}$ is the adjoint of the linear map $L_{x}$.

Let us denote by $\left\{\tau_{1}, \ldots, \tau_{N-1}\right\}$ an orthonormal base for $\pi_{x}^{S}$; thus, $\left\{\tau_{1}, \ldots, \tau_{N-1}, \nu^{S}(x)\right\}$ is an orthonormal base for $\mathbb{R}^{N}$. Denoting by $\left(a_{i j}\right)$ the $k \times k$ matrix representing the linear map $L_{x} \circ L_{x}^{*}$ with respect to the standard base in $\mathbb{R}^{k}$, we get that

$$
a_{i j}=\sum_{h=1}^{N-1}\left\langle\tau_{h}, e_{i}\right\rangle\left\langle\tau_{h}, e_{j}\right\rangle \quad \text { for all } i, j=1, \ldots, k
$$

Writing, for all $i, j, e_{i}=\sum_{h=1}^{N-1}\left\langle\tau_{h}, e_{i}\right\rangle \tau_{h}+\left\langle\nu^{S}(x), e_{i}\right\rangle \nu^{S}(x)$, we get

$$
\delta_{i j}=\left\langle e_{i}, e_{j}\right\rangle=\sum_{h=1}^{N-1}\left\langle\tau_{h}, e_{i}\right\rangle\left\langle\tau_{h}, e_{j}\right\rangle+\nu_{i}^{S} \nu_{j}^{S}
$$

hence

$$
a_{i j}=\delta_{i j}-\nu_{i}^{S} \nu_{j}^{S} \quad \text { for all } i, j=1, \ldots, k
$$

From this formula it follows that

$$
C_{k} L_{x}=\sqrt{\operatorname{det}(I-\widetilde{\nu} \otimes \widetilde{\nu})},
$$

where $I$ is the $k \times k$ identity matrix and $\widetilde{\nu}=\left(\nu_{1}^{S}(x), \ldots, \nu_{k}^{S}(x)\right)$. The assertion then follows by observing that

$$
\operatorname{det}(I-\widetilde{\nu} \otimes \widetilde{\nu})=1-|\widetilde{\nu}|^{2}=\sum_{i=k+1}^{N}\left|\nu_{i}^{S}\right|^{2} .
$$

For a general survey on measures and BV-functions we refer to $[2,12,13,19,22]$. 


\section{Sections of BV-Functions}

In this section we state some fine properties of BV-functions, which will be needed in the sequel.

Lemma 4.1. Let $g: \Omega \rightarrow \mathbb{R}$ be a Borel function. Assume that $g \in L_{\mathrm{loc}}^{1}(\Omega)$ and set

$$
G^{*}:=\left\{\left(x^{\prime}, y\right) \in \Omega \backslash S_{g}: \lim _{\varepsilon \rightarrow 0^{+}} f_{Q^{\prime}\left(x^{\prime}, \varepsilon\right)}\left|g\left(z^{\prime}, y\right)-g^{*}\left(x^{\prime}, y\right)\right| \mathrm{d} z^{\prime}=0\right\}
$$

where $Q^{\prime}\left(x^{\prime}, \varepsilon\right):=x^{\prime}+\varepsilon Q^{\prime}$ and $Q^{\prime}=(-1 / 2,1 / 2)^{N-1}$. Then $G^{*}$ is a Borel set.

Proof. Notice that $G^{*}=G_{1} \cap G_{2}$, where

$$
G_{1}:=\left\{\left(x^{\prime}, y\right) \in \Omega \backslash S_{g}: \text { there exists } z \in \mathbb{R} \text { such that } \lim _{\varepsilon \rightarrow 0^{+}} f_{Q^{\prime}\left(x^{\prime}, \varepsilon\right)}\left|g\left(z^{\prime}, y\right)-z\right| \mathrm{d} z^{\prime}=0\right\}
$$

and

$$
G_{2}:=\left\{\left(x^{\prime}, y\right) \in \Omega \backslash S_{g}: \lim _{\varepsilon \rightarrow 0^{+}} f_{Q^{\prime}\left(x^{\prime}, \varepsilon\right)} g\left(z^{\prime}, y\right) \mathrm{d} z^{\prime}=g^{*}\left(x^{\prime}, y\right)\right\} .
$$

We claim that $G_{1}$ is a Borel set. In fact, consider a dense sequence $\left\{q_{i}\right\} \subset \mathbb{R}$ and, for every $i, j \in \mathbb{N}$, set

$$
G_{i j}=\left\{\left(x^{\prime}, y\right) \in \Omega: \limsup _{\varepsilon \rightarrow 0^{+}} f_{Q^{\prime}\left(x^{\prime}, \varepsilon\right)}\left|g\left(z^{\prime}, y\right)-q_{i}\right| \mathrm{d} z^{\prime}<\frac{1}{j}\right\}
$$

It is not difficult to check that if $h: \mathbb{R}^{N} \rightarrow \mathbb{R}$ is a locally summable Borel function, then also $\left(x^{\prime}, y\right) \mapsto$ $f_{Q^{\prime}\left(x^{\prime}, \varepsilon\right)} h\left(z^{\prime}, y\right) \mathrm{d} z^{\prime}$ is a Borel function for any $\varepsilon>0$. From this fact we get immediately that each $G_{i j}$ is a Borel set, and since

$$
G_{1}=\bigcap_{j=1}^{\infty} \bigcup_{i=1}^{\infty} G_{i j}
$$

our claim follows. On the other hand, since for any $\varepsilon>0$ the function $\left(x^{\prime}, y\right) \mapsto f_{Q^{\prime}\left(x^{\prime}, \varepsilon\right)} g\left(z^{\prime}, y\right) \mathrm{d} z^{\prime}$ is Borel, we easily get that also $G_{2}$ is a Borel set. This concludes the proof.

Lemma 4.2. ([2], Th. 3.108) Let $g \in \mathrm{BV}(\Omega)$ be a given function. Then, for $\mathcal{L}^{N-1}$-almost every $x^{\prime} \in \pi_{N-1}(\Omega)$, the function $g_{x^{\prime}}$ belongs to $\mathrm{BV}\left(\Omega_{x^{\prime}}\right)$. Moreover $J_{g_{x^{\prime}}}=\left(J_{g}\right)_{x^{\prime}}$ and $\left(g^{*}\right)_{x^{\prime}}(y)=\left(g_{x^{\prime}}\right)^{*}(y)$ for every $y \in \Omega_{x^{\prime}} \backslash\left(J_{g}\right)_{x^{\prime}}$. In particular, if $\left(J_{g}\right)_{x^{\prime}}=\emptyset$, then $\left(g^{*}\right)_{x^{\prime}}(y)=\left(g_{x^{\prime}}\right)^{*}(y)$ for every $y \in \Omega_{x^{\prime}}$ and both functions $\left(g^{*}\right)_{x^{\prime}}$ and $\left(g_{x^{\prime}}\right)^{*}$ are continuous in $\Omega_{x^{\prime}}$.

Lemma 4.3. Let $g \in \operatorname{BV}(\Omega)$ be a given function. Then, for $\mathcal{L}^{1}$-almost every $y \in \pi_{1}(\Omega)$, the function $g_{y}$ belongs to $\mathrm{BV}\left(\Omega_{y}\right)$.

Proof. If $N=2$, the property follows by Lemma 4.2. If $N>2$, the property can be easily obtained following the proof of [2], Theorem 3.103.

In the next two lemmas we assume $N>2$. For every $x=\left(x^{\prime}, y\right) \in \mathbb{R}^{N}$, we set

$$
\hat{x}_{i}^{\prime}=\left(x_{1}, \ldots, x_{i-1}, x_{i+1}, \ldots, x_{N-1}\right) \in \mathbb{R}^{N-2}
$$

and write, for the sake of simplicity, $x=\left(x_{i}, \hat{x}_{i}^{\prime}, y\right)$. Moreover, if $E$ is a given set, with $E_{\hat{x}_{i}^{\prime} y}$ we denote $E_{x_{\nu}^{\perp}}$, with $\nu=\mathrm{e}_{i}$; a similar notation will be used also for functions. 
Lemma 4.4. Let $g \in \mathrm{BV}(\Omega)$ be a given function. Then there exists a set $N_{0} \subset \mathbb{R}$ with $\mathcal{L}^{1}\left(N_{0}\right)=0$ with the following property: for every $y \in \mathbb{R} \backslash N_{0}, g_{y} \in \mathrm{BV}\left(\Omega_{y}\right)$ and, for every $i=1, \ldots, N-1$, there exists a set $N^{i y} \subset \mathbb{R}^{N-2}$ with $\mathcal{L}^{N-2}\left(N^{i y}\right)=0$ such that, for every $\hat{x}_{i}^{\prime} \in \mathbb{R}^{N-2} \backslash N^{i y}$ we have that $g_{\hat{x}_{i}^{\prime} y} \in \mathrm{BV}\left(\Omega_{\hat{x}_{i}^{\prime} y}\right)$ and

$$
\begin{gathered}
\left(S_{g_{y}}\right)_{\hat{x}_{i}^{\prime}}=J_{{\hat{x^{\prime}}}_{i}^{\prime}} \\
{\left[\left(g_{y}\right)^{*}\right]_{\hat{x}_{i}^{\prime}}\left(x_{i}\right)=\left(g_{\hat{x}_{i}^{\prime} y}\right)^{*}\left(x_{i}\right) \quad \text { for every } x_{i} \in \Omega_{\hat{x}_{i}^{\prime} y} \backslash J_{\hat{x}_{i}^{\prime} y} .}
\end{gathered}
$$

Proof. For the sake of simplicity, assume $\Omega=\mathbb{R}^{N}$. By Lemma 4.3 there exists $N_{0} \subset \mathbb{R}$ with $\mathcal{L}^{1}\left(N_{0}\right)=0$ such that for every $y \in \mathbb{R} \backslash N_{0}$ we have that $g_{y} \in B V\left(\mathbb{R}^{N-1}\right), J_{g_{y}}$ is a countably $\mathcal{H}^{N-2}$-rectifiable set and $\mathcal{H}^{N-2}\left(S_{g_{y}} \backslash J_{g_{y}}\right)=0$. Moreover, by Theorem 3.2, we have that, for every $i=1, \ldots, N-1$,

$$
0=\int_{S_{g_{y}} \backslash J_{g_{y}}}\left|\left\langle\nu^{S_{g_{y}}}, e_{i}\right\rangle\right| \mathrm{d} \mathcal{H}^{N-2}=\int_{\mathbb{R}^{N-2}} \mathcal{H}^{0}\left(\left(S_{g_{y}} \backslash J_{g_{y}}\right)_{\hat{x}_{i}^{\prime}}\right) \mathrm{d} \hat{x}_{i}^{\prime}
$$

so that there exists a set $N_{1}^{i y} \subset \mathbb{R}^{N-2}$ with $\mathcal{L}^{N-2}\left(N_{1}^{i y}\right)=0$, such that for every $\hat{x}_{i}^{\prime} \in \mathbb{R}^{N-2} \backslash N_{1}^{i y}$ we have $\left(S_{g_{y}} \backslash J_{g_{y}}\right)_{\hat{x}_{i}^{\prime}}=\emptyset ;$ i.e.,

$$
\left(S_{g_{y}}\right)_{\hat{x}_{i}^{\prime}}=\left(J_{g_{y}}\right)_{\hat{x}_{i}^{\prime}} \cdot
$$

Moreover, by Lemma 4.2 applied to $g_{y}$, we have that for every $y \in \mathbb{R} \backslash N_{0}$ and for every $i=1, \ldots, N-1$, there exists a set $N_{2}^{i y} \subset \mathbb{R}^{N-2}$ with $\mathcal{L}^{N-2}\left(N_{2}^{i y}\right)=0$, such that for every $\hat{x}_{i}^{\prime} \in \mathbb{R}^{N-2} \backslash N_{2}^{i y}$ we have that $g_{\hat{x}_{i}^{\prime} y} \in B V(\mathbb{R})$ and

$$
\begin{aligned}
\left(J_{g_{y}}\right)_{\hat{x}_{i}^{\prime}} & =J_{g_{\hat{x}_{i}^{\prime} y}} . \\
{\left[\left(g_{y}\right)^{*}\right]_{\hat{x}_{i}^{\prime}}\left(x_{i}\right) } & =\left(g_{\hat{x}_{i}^{\prime} y}\right)^{*}\left(x_{i}\right) \quad \text { for every } x_{i} \in \mathbb{R} \backslash J_{{\hat{x_{i}^{\prime}}}_{i}^{\prime}} .
\end{aligned}
$$

Finally, for every $y \in \mathbb{R} \backslash N_{0}$ and for every $i=1, \ldots, N-1$, set $N^{i y}=N_{1}^{i y} \cup N_{2}^{i y} \subset \mathbb{R}^{N-2}$, so that $\mathcal{L}^{N-2}\left(N^{i y}\right)=0$ and for every $\hat{x}_{i}^{\prime} \in \mathbb{R}^{N-2} \backslash N^{i y}$ we have that (4.3), (4.4) and (4.5) hold. Hence the assertion follows.

Lemma 4.5. Let $g \in \mathrm{BV}(\Omega)$ be a function which is approximately continuous in $\mathcal{H}^{N-1}$-almost every point of $\Omega$. Then there exists a set $M_{0} \subset \mathbb{R}$ with $\mathcal{L}^{1}\left(M_{0}\right)=0$ with the following property: for every $y \in \mathbb{R} \backslash M_{0}, g_{y} \in \mathrm{BV}\left(\Omega_{y}\right)$ and, for every $i=1, \ldots, N-1$, there exists a set $M^{i y} \subset \mathbb{R}^{N-2}$ with $\mathcal{L}^{N-2}\left(M^{i y}\right)=0$ such that, for every $\hat{x}_{i}^{\prime} \in \mathbb{R}^{N-2} \backslash M^{i y}$ we have that $g_{\hat{x}_{i}^{\prime} y} \in \mathrm{BV}\left(\Omega_{\hat{x}_{i}^{\prime} y}\right)$ and

$$
\begin{aligned}
& J_{\hat{x}_{\hat{x}^{\prime} y}}=\left(S_{g}\right)_{\hat{x}_{i}^{\prime} y}=\left(J_{g}\right)_{\hat{x}_{i}^{\prime} y}=\emptyset, \\
& \left(g_{\hat{x}_{i}^{\prime} y}\right)^{*}\left(x_{i}\right)=\left(g^{*}\right)_{\hat{x}_{i}^{\prime} y}\left(x_{i}\right) \quad \text { for every } x_{i} \in \Omega_{\hat{x}_{i}^{\prime} y}
\end{aligned}
$$

and both functions are continuous.

Proof. As before, we assume for simplicity $\Omega=\mathbb{R}^{N}$. By assumption we have that $\mathcal{H}^{N-1}\left(S_{g}\right)=0$, thus from Theorem 3.2 we get that for every $i=1, \ldots, N-1$

$$
0=\int_{S_{g}}\left|\left\langle\nu^{S_{g}}, e_{i}\right\rangle\right| \mathrm{d} \mathcal{H}^{N-1}=\int_{\mathbb{R}^{N-1}} \mathcal{H}^{0}\left(\left(S_{g}\right)_{\hat{x}_{i}^{\prime} y}\right) \mathrm{d} \hat{x}_{i}^{\prime} \mathrm{d} y .
$$

Therefore there exists $M_{1}^{i} \subset \mathbb{R}^{N-1}$, with $\mathcal{L}^{N-1}\left(M_{1}^{i}\right)=0$, such that for every $\left(\hat{x}_{i}^{\prime}, y\right) \in \mathbb{R}^{N-1} \backslash M_{1}^{i}$ we have $\left(S_{g}\right)_{\hat{x}_{i}^{\prime} y}=\emptyset$. Set

$$
M_{1}=\left\{y \in \mathbb{R}: \mathcal{L}^{N-2}\left(\left(M_{1}^{i}\right)_{y}\right)>0 \text { for at least one index } i=1, \ldots, N-1\right\} .
$$

Then, by Fubini's theorem

$$
0=\mathcal{L}^{N-1}\left(M_{1}^{i}\right)=\int_{\mathbb{R}} \mathcal{L}^{N-2}\left(\left(M_{1}^{i}\right)_{y}\right) \mathrm{d} y
$$


so that $\mathcal{L}^{N-2}\left(\left(M_{1}^{i}\right)_{y}\right)=0$ for a.e. $y \in \mathbb{R} ;$ i.e., $\mathcal{L}^{1}\left(M_{1}\right)=0$. Moreover, for every $y \in \mathbb{R} \backslash M_{1}$ and every $\hat{x}_{i}^{\prime} \in \mathbb{R}^{N-2} \backslash\left(M_{1}^{i}\right)_{y}$, recalling that $\left(J_{g}\right)_{\hat{x}_{i}^{\prime} y} \subseteq\left(S_{g}\right)_{\hat{x}_{i}^{\prime} y}$, we have

$$
\left(S_{g}\right)_{\hat{x}_{i}^{\prime} y}=\left(J_{g}\right)_{\hat{x}_{i}^{\prime} y}=\emptyset
$$

By Lemma 4.2 we have that for every $i=1, \ldots, N-1$, there exists a set $M_{2}^{i} \subset \mathbb{R}^{N-1}$ with $\mathcal{L}^{N-1}\left(M_{2}^{i}\right)=0$, such that for every $\left(\hat{x}_{i}^{\prime}, y\right) \in \mathbb{R}^{N-1} \backslash M_{2}^{i}$ we have that $g_{\hat{x}_{i}^{\prime} y} \in B V(\mathbb{R})$ and

$$
\begin{gathered}
J_{g_{\hat{x}_{i}^{\prime} y}}=\left(J_{g}\right)_{\hat{x}_{i}^{\prime} y}, \\
\left(g^{*}\right)_{\hat{x}_{i}^{\prime} y}\left(x_{i}\right)=\left(g_{\hat{x}_{i}^{\prime} y}\right)^{*}\left(x_{i}\right) \quad \text { for every } x_{i} \in \mathbb{R} \backslash\left(J_{g}\right)_{\hat{x}_{i}^{\prime} y} .
\end{gathered}
$$

Set

$$
M_{2}=\left\{y \in \mathbb{R}: \mathcal{L}^{N-2}\left(\left(M_{2}^{i}\right)_{y}\right)>0 \text { for at least one index } i=1, \ldots, N-1\right\} .
$$

As before, Fubini's theorem implies that $\mathcal{L}^{1}\left(M_{2}\right)=0$. Thus for any $y \in \mathbb{R} \backslash M_{2}$ and any $\hat{x}_{i}^{\prime} \in \mathbb{R}^{N-2} \backslash\left(M_{2}^{i}\right)_{y}$ we obtain that (4.9) and (4.10) hold. Finally, set $M_{0}=M_{1} \cup M_{2} \subset \mathbb{R}$ and $M^{i y}=\left(M_{1}^{i}\right)_{y} \cup\left(M_{2}^{i}\right)_{y}$, so that $\mathcal{L}^{1}\left(M_{0}\right)=0$ and, for every $y \in \mathbb{R} \backslash M_{0}, \mathcal{L}^{N-2}\left(M^{i y}\right)=0$. Moreover, for every $y \in \mathbb{R} \backslash M_{0}$, by (4.8), (4.9) and (4.10) we have

$$
\begin{aligned}
& \emptyset=\left(S_{g}\right)_{\hat{x}_{i}^{\prime} y}=\left(J_{g}\right)_{\hat{x}_{i}^{\prime} y}=J_{g_{\hat{x}_{i}^{\prime} y}}, \\
&\left(g^{*}\right)_{\hat{x}_{i}^{\prime} y}\left(x_{i}\right)=\left(g_{\hat{x}_{i}^{\prime} y}\right)^{*}\left(x_{i}\right) \quad \text { for every } x_{i} \in \mathbb{R}
\end{aligned}
$$

and by Lemma 4.2 both functions are continuous.

Next theorem states that given a $\mathcal{H}^{N-1}$-a.e. approximately continuous $B V$ function $g$, its $(N-1)$-dimensional sections are still $\mathcal{H}^{N-1}$-a.e. approximately continuous along a countably $\mathcal{H}^{N-1}$-rectifiable set whose normals are "never" orthogonal to the direction in which the sections are taken.

Theorem 4.6. Let $g \in \operatorname{BV}(\Omega)$ be a Borel function which is approximately continuous in $\mathcal{H}^{N-1}$-almost every point of $\Omega$. Set

$$
G=\left\{\left(x^{\prime}, y\right) \in \Omega \backslash S_{g}: \lim _{\varepsilon \rightarrow 0^{+}} \int_{Q^{\prime}\left(x^{\prime}, \varepsilon\right)}\left|g\left(z^{\prime}, y\right)-g\left(x^{\prime}, y\right)\right| \mathrm{d} z^{\prime}=0\right\} .
$$

Let $S \subset \Omega$ be a countably $\mathcal{H}^{N-1}$-rectifiable set such that $\mathcal{H}^{N-1}\left(\left\{x \in S: \nu^{S}(x)= \pm e_{N}\right\}\right)=0$. Then $\mathcal{H}^{N-1}(S \backslash G)=0$.

Proof. Again, we assume for simplicity that $\Omega=\mathbb{R}^{N}$.

Since $g$ is approximately continuous $\mathcal{H}^{N-1}$-a.e., the thesis will be achieved if we prove that $\mathcal{H}^{N-1}\left(S \backslash G^{*}\right)=0$, where $G^{*}$ is the set defined in Lemma 4.1. To this aim, let us first assume that $N>2$.

Following the notation used in Lemmas 4.4 and 4.5 let us take $y \in \mathbb{R} \backslash\left(N_{0} \cup M_{0}\right)$ and for every $i=1, \ldots, N-1$, $\hat{x}_{i}^{\prime} \in \mathbb{R}^{N-2} \backslash\left(N^{i y} \cup M^{i y}\right)$. Then by (4.1) and (4.6) we obtain

$$
\left(S_{g}\right)_{\hat{x}_{i}^{\prime} y}=\left(S_{g_{y}}\right)_{\hat{x}_{i}^{\prime}}=\emptyset
$$

so that, for every $x_{i} \in \mathbb{R}$ we have that $x_{i} \notin\left(S_{g}\right)_{\hat{x}_{i}^{\prime} y}$, (i.e., $\left.x=\left(x_{i}, \hat{x}_{i}^{\prime}, y\right) \notin S_{g}\right)$ and $x_{i} \notin\left(S_{g_{y}}\right)_{\hat{x}_{i}^{\prime}}\left(i . e ., x^{\prime}=\left(x_{i}, \hat{x}_{i}^{\prime}\right) \notin\right.$ $S_{g_{y}}$ ). Hence, $x$ is a point where $g$ has an approximate limit and $x^{\prime}$ is a point where $g_{y}$ has an approximate limit. Moreover by (4.2) and (4.7) it follows that $\left(g_{y}\right)^{*}\left(x^{\prime}\right)=\left(g^{*}\right)_{\hat{x}_{i}^{\prime} y}\left(x_{i}\right)=g^{*}(x)$, i.e.,

$$
\lim _{\varepsilon \rightarrow 0^{+}} f_{Q^{\prime}\left(x^{\prime}, \varepsilon\right)}\left|g\left(z^{\prime}, y\right)-g^{*}(x)\right| \mathrm{d} z^{\prime}=\lim _{\varepsilon \rightarrow 0^{+}} f_{Q^{\prime}\left(x^{\prime}, \varepsilon\right)}\left|g\left(z^{\prime}, y\right)-\left(g_{y}\right)^{*}\left(x^{\prime}\right)\right| \mathrm{d} z^{\prime}=0
$$

which implies that $x \in G^{*}$; i.e., $x_{i} \in G_{\hat{x}_{i}^{\prime} y}^{*}$. In particular we obtain that, for every $y \in \mathbb{R} \backslash\left(N_{0} \cup M_{0}\right)$, every $i=1, \ldots, N-1$ and every $\hat{x}_{i}^{\prime} \in \mathbb{R}^{N-2} \backslash\left(N^{i y} \cup M^{i y}\right)$

$$
G_{\hat{x}_{i}^{\prime} y}^{*}=\mathbb{R}
$$


Since $\left|\nu^{S_{y}}\right|=1$, from Theorem 3.2 and (4.11) we obtain

$$
\begin{aligned}
\mathcal{H}^{N-2}\left(\left(S \backslash G^{*}\right)_{y}\right) & =\sum_{i=1}^{N-1} \int_{\left(S \backslash G^{*}\right)_{y}}\left|\left\langle\nu^{S_{y}}, e_{i}\right\rangle\right|^{2} \mathrm{~d} \mathcal{H}^{N-2} \leq \sum_{i=1}^{N-1} \int_{\left(S \backslash G^{*}\right)_{y}}\left|\left\langle\nu^{S_{y}}, e_{i}\right\rangle\right| \mathrm{d} \mathcal{H}^{N-2} \\
& =\sum_{i=1}^{N-1} \int_{\mathbb{R}^{N-2}} \mathcal{H}^{0}\left(\left[\left(S \backslash G^{*}\right)_{y}\right]_{\hat{x}_{i}^{\prime}}\right) \mathrm{d} \hat{x}_{i}^{\prime}=\sum_{i=1}^{N-1} \int_{\mathbb{R}^{N-2}} \mathcal{H}^{0}\left(\left(S \backslash G^{*}\right)_{\hat{x}_{i}^{\prime} y}\right) \mathrm{d} \hat{x}_{i}^{\prime}=0
\end{aligned}
$$

which implies $\mathcal{H}^{N-2}\left(\left(S \backslash G^{*}\right)_{y}\right)=0$ for every $y \in \mathbb{R} \backslash\left(N_{0} \cup M_{0}\right)$. Finally, using again Theorem 3.2, we obtain

$$
\int_{S \backslash G^{*}} \sqrt{1-\left|\left\langle\nu^{S}, e_{N}\right\rangle\right|^{2}} \mathrm{~d} \mathcal{H}^{N-1}=\int_{\mathbb{R}} \mathcal{H}^{N-2}\left(\left(S \backslash G^{*}\right)_{y}\right) \mathrm{d} y=0 .
$$

Therefore, taking into account the assumption made on $S$, we have $\mathcal{H}^{N-1}\left(S \backslash G^{*}\right)=0$.

If $N=2$, we apply the coarea formula (3.1) again, thus getting

$$
\int_{S \backslash G^{*}}\left|\left\langle\nu^{S}, e_{1}\right\rangle\right| \mathrm{d} \mathcal{H}^{1}=\int_{\mathbb{R}} \mathcal{H}^{0}\left(\left(S \backslash G^{*}\right)_{y}\right) \mathrm{d} y=0,
$$

where the last equality holds since $\mathcal{H}^{1}\left(S_{g}\right)=0$ implies $\left(J_{g}\right)_{y}=\emptyset$ for $\mathcal{L}^{1}$-almost every $y \in \pi_{1}(\Omega)$ and, by Lemma 4.2 , $\left(g^{*}\right)_{y}(x)=\left(g_{y}\right)^{*}(x)$ for all $x \in \Omega_{y}$ and for $\mathcal{L}^{1}$-almost every $y \in \pi_{1}(\Omega)$. Hence, the assertion follows.

Remark 4.7. Clearly, Theorem 4.6 still holds if we replace $\mathrm{e}_{N}$ by a generic direction $\nu$. More precisely, given any direction $\nu \in \mathbb{S}^{N-1}$, set

$$
G_{\nu}=\left\{x=\left(x_{\nu}^{\perp}, x_{\nu}\right) \in \Omega \backslash S_{g}: \lim _{\varepsilon \rightarrow 0^{+}} f_{Q_{\nu}^{\perp}(x, \varepsilon)}\left|g\left(z_{\nu}^{\perp}, x_{\nu}\right)-g\left(x_{\nu}^{\perp}, x_{\nu}\right)\right| \mathrm{d} z_{\nu}^{\perp}=0\right\}
$$

where $Q_{\nu}^{\perp}(x, \varepsilon)=\pi_{\nu^{\perp}}\left(x+\varepsilon Q_{\nu}\right), Q_{\nu}=R_{\nu}(-1 / 2,1 / 2)^{N}$ and $R_{\nu}$ denotes a rotation such that $R_{\nu} \mathrm{e}_{N}=\nu$. Then $\mathcal{H}^{N-1}\left(S \backslash G_{\nu}\right)=0$ for every countably $\mathcal{H}^{N-1}$-rectifiable subset $S$ of $\Omega$, such that $\mathcal{H}^{N-1}\left(\left\{x \in S: \nu^{S}(x)= \pm \nu\right\}\right)=0$.

\section{Setting of the Problem}

Let $f: \Omega \times \mathbb{R} \times \mathbb{R}^{N} \rightarrow \mathbb{R}$ be a Borel function satisfying the following conditions:

$$
\begin{cases}\text { (i) } & f(\cdot, s, \xi) \in W^{1,1}(\Omega) \text {, for every }(s, \xi) \in \mathbb{R} \times \mathbb{R}^{N} ; \\ \text { (ii) } f(\cdot, s, \xi) \text { is approximately continuous } \mathcal{H}^{N-1} \text {-a.e. in } \Omega \text {, for every }(s, \xi) \in \mathbb{R} \times \mathbb{R}^{N} ; \\ \text { (iii) for every bounded set } B \subset \mathbb{R} \times \mathbb{R}^{N} \text { there exists a constant } L(B) \text { such that } \\ \\ \int_{\Omega}\left|\nabla_{x} f\right|(x, s, \xi) \mathrm{d} x<L(B) \quad \forall(s, \xi) \in B .\end{cases}
$$

Remark 5.1. Notice that assumption (ii) of (5.1) seems redundant, since every $W^{1,1}$-function admits a $\mathcal{H}^{N-1}$-a.e. approximately continuous representative. Moreover, the functional in (5.5) is clearly not affected by the choice of the representative. However, functional (5.6) does depend on the particular representative chosen. Therefore, the representation formula provided by Theorem 6.1 below does not hold if we take a representative of $f$ not satisfying (ii).

We will assume that

$$
\begin{aligned}
& f(x, s, \cdot) \quad \text { is convex for every }(x, s) \in \Omega \times \mathbb{R} ; \\
& \left|f(x, s, \xi)-f\left(x, s_{0}, \xi\right)\right| \leq \Lambda(1+|\xi|)\left|s-s_{0}\right| \quad \text { for every }(x, s, \xi),\left(x, s_{0}, \xi\right) \in \Omega \times \mathbb{R} \times \mathbb{R}^{N} \\
& 0 \leq f(x, s, \xi) \leq \Lambda(1+|\xi|) \quad \text { for every }(x, s, \xi) \in \Omega \times \mathbb{R} \times \mathbb{R}^{N},
\end{aligned}
$$


for some positive $\Lambda$. From (5.2) and (5.4), it follows that $f$ is Lipschitz continuous in the last variable, uniformly with respect to $(x, s)$.

For every $A \in \mathcal{A}(\Omega)$ and every $u \in \operatorname{BV}(\Omega)$, we define

$$
F(u, A)= \begin{cases}\int_{A} f(x, u, \nabla u) \mathrm{d} x & \text { if } u \in W^{1,1}(\Omega) \\ +\infty & \text { if } u \in \mathrm{BV}(\Omega) \backslash W^{1,1}(\Omega) .\end{cases}
$$

Our aim is to prove an integral representation theorem for the relaxation $\bar{F}$ of $F$, with respect to the $L^{1}$-topology. We recall that the relaxation of $F$ is the greatest lower semicontinuous functional not greater than $F$; i.e.,

$$
\bar{F}(u, \Omega):=\inf \left\{\liminf _{n \rightarrow+\infty} F\left(u_{n}, \Omega\right): u_{n} \in W^{1,1}(\Omega), u_{n} \rightarrow u \text { in } L^{1}(\Omega)\right\} .
$$

Among the main properties of the relaxation, we recall the following ones:

(i) for every $A \in \mathcal{A}(\Omega), \bar{F}(\cdot, A)$ is lower semicontinuous with respect to the $L^{1}$-topology;

(ii) for every $A \in \mathcal{A}(\Omega), \bar{F}(\cdot, A)$ is local; i.e., for every $u, v \in \operatorname{BV}(\Omega)$, with $u=v$ on $A, \bar{F}(u, A)=\bar{F}(v, A)$;

(iii) for every $u \in \operatorname{BV}(\Omega), \bar{F}(u, \cdot)$ is a $\sigma$-additive measure on $\mathcal{B}(\Omega)$.

For other properties of the relaxation we refer to $[4,6,10,11]$.

We set, for every $A \in \mathcal{A}(\Omega)$ and every $u \in B V(\Omega)$,

$$
\mathcal{F}(u, A)=\int_{A} f(x, u, \nabla u) \mathrm{d} x+\int_{A} f^{\infty}\left(x, \widetilde{u}, \frac{D^{c} u}{\left|D^{c} u\right|}\right) \mathrm{d}\left|D^{c} u\right|+\int_{J_{u} \cap A}\left(\int_{u^{-}(x)}^{u^{+}(x)} f^{\infty}\left(x, s, \nu_{u}\right) \mathrm{d} s\right) \mathrm{d} \mathcal{H}^{N-1}(x),
$$

where $f^{\infty}: \Omega \times \mathbb{R} \times \mathbb{R}^{N} \rightarrow \mathbb{R}$ is the so-called recession function of $f$, defined by

$$
f^{\infty}(x, s, \xi)=\lim _{t \rightarrow+\infty} \frac{f(x, s, t \xi)}{t}=\sup _{t>0} \frac{f(x, s, t \xi)-f(x, s, 0)}{t} .
$$

Notice that assumptions (5.2) and (5.4) imply that the limit in (5.7) exists for every $(x, s, \xi) \in \Omega \times \mathbb{R} \times \mathbb{R}^{N}$ (since the function $t \mapsto \frac{f(x, s, t \xi)-f(x, s, 0)}{t}$ is increasing). Moreover, the function $f^{\infty}$ is convex and positively homogeneous of degree one in the last variable and, as a consequence of definition (5.7), we have that

$$
\frac{f(x, s, t \xi)}{t} \leq f^{\infty}(x, s, \xi)+\frac{f(x, s, 0)}{t} \quad \text { for all } t>0 .
$$

Notice also that $f^{\infty}$ is a Borel function in $\Omega \times \mathbb{R} \times \mathbb{R}^{N}$. Thus, the functional $\mathcal{F}$ in (5.6) is well defined. By the assumptions made on $f$, it follows that

$$
\begin{array}{ll}
0 \leq f^{\infty}(x, s, \xi) \leq \Lambda|\xi| & \text { for every }(x, s, \xi) \in \Omega \times \mathbb{R} \times \mathbb{R}^{N}, \\
\left|f^{\infty}(x, s, \xi)-f^{\infty}\left(x, s_{0}, \xi\right)\right| \leq \Lambda|\xi|\left|s-s_{0}\right| & \text { for every }(x, s, \xi),\left(x, s_{0}, \xi\right) \in \Omega \times \mathbb{R} \times \mathbb{R}^{N} .
\end{array}
$$

In the sequel, we will assume also that

$$
f^{\infty}(\cdot, s, \xi) \in \operatorname{BV}(\Omega) \quad \text { for every }(s, \xi) \in \mathbb{R} \times \mathbb{R}^{N},
$$

and that, for any $(s, \xi) \in \mathbb{R} \times \mathbb{R}^{N}$,

$$
f^{\infty}(\cdot, s, \xi) \text { is approximately continuous for } \mathcal{H}^{N-1} \text {-a.e. } x \in \Omega \text {. }
$$


Remark 5.2. Note that (5.11), (5.12) trivially follow from $(5.1)$ when $f(x, s, \cdot)$ is positively 1-homogeneous (since in this case $f^{\infty}$ coincides with $f$ ) and when $f(x, s, \xi)=a(x, s) b(\xi)$ or $f(x, s, \xi)=a(x) b(s, \xi)$ (since in this case the dependence on $x$ is not involved in the limit (5.7)). However, in general, property (5.12) is not a consequence of (5.1)-(5.4), as the following example shows.

Example 5.3. Let $f:(-1,1) \times \mathbb{R} \rightarrow[0,+\infty)$ be a function defined by

$$
f(x, \xi)= \begin{cases}\frac{5}{2}|\xi|-\frac{1}{2 x} & \text { if } \quad x \geq \frac{1}{|\xi|} \\ \frac{x|\xi|^{2}}{2}+\frac{3}{2}|\xi| & \text { if } \quad 0 \leq x \leq \frac{1}{|\xi|} \\ \frac{3}{2}|\xi| & \text { if } \quad x \leq 0\end{cases}
$$

if $\xi \neq 0$ and $f(x, 0)=0$ for every $x \in(-1,1)$. It is easy to check that $f$ is a Lipschitz function with respect to $x$ satisfying (5.1)-(5.4). Nevertheless, condition (5.12) does not hold, since

$$
f^{\infty}(x, \xi)=\left\{\begin{array}{lll}
\frac{5}{2}|\xi| & \text { if } & x>0 \\
\frac{3}{2}|\xi| & \text { if } & x \leq 0 .
\end{array}\right.
$$

We believe that assumptions (5.1), (5.2) and (5.4) do not imply that $x \mapsto f^{\infty}(\cdot, \xi)$ is a $B V$ function. However, next proposition states that under further assumptions on $f$, then the recession function $f^{\infty}$ necessarily satisfies (5.11) and (5.12).

Proposition 5.4. Let $f: \Omega \times \mathbb{R} \times \mathbb{R}^{N} \rightarrow \mathbb{R}$ be a function satisfying (5.1), (5.2) and (5.4). Assume that

- (i) $f_{\xi}(\cdot, s, \xi)$ is weakly differentiable in $\Omega$ for all $(s, \xi)$ and that

$$
\int_{\Omega}\left|\nabla_{x} f_{\xi}(x, s, \xi)\right| \mathrm{d} x \leq c_{0} \quad \text { for all }(s, \xi) \in \mathbb{R} \times \mathbb{R}^{N}
$$

Then $f^{\infty}$ satisfies (5.11). Moreover, if

- (ii) for every $\left(x_{0}, s\right) \in \Omega \times \mathbb{R}$ and for every $\varepsilon>0$ there exist $\delta>0$ and $L>0$ such that

$$
\left|f^{\infty}(x, s, \xi)-\frac{f(x, s, t \xi)}{t}\right| \leq \varepsilon\left(1+\frac{f(x, s, t \xi)}{t}\right)
$$

for any $x \in \Omega$, with $\left|x-x_{0}\right| \leq \delta$, any $\xi \in \mathbb{R}^{N}$ and any $t>L$, then $f^{\infty}$ satisfies also (5.12).

Proof. Fix $s \in \mathbb{R}$ and $\varphi \in C_{0}^{1}\left(\Omega, \mathbb{R}^{N}\right)$ and set $\psi_{s}(\xi)=\int_{\Omega}\left\langle\nabla_{x} f(x, s, \xi), \varphi(x)\right\rangle \mathrm{d} x$. From assumption (i) we get easily that $\left|\nabla \psi_{s}(\xi)\right| \leq c_{0}\|\varphi\|_{\infty}$ for all $(s, \xi) \in \mathbb{R} \times \mathbb{R}^{N}$. Hence $\psi_{s}$ is Lipschitz continuous and $\operatorname{Lip}\left(\psi_{s}\right) \leq c_{0}\|\varphi\|_{\infty}$. Therefore, from (5.4) and (5.1), we get that for all $(s, \xi)$

$$
\begin{aligned}
\int_{\Omega} f^{\infty}(x, s, \xi) \operatorname{div} \varphi(x) \mathrm{d} x & =\lim _{h \rightarrow \infty} \int_{\Omega} \frac{f(x, s, h \xi)}{h} \operatorname{div} \varphi(x) \mathrm{d} x=\lim _{h \rightarrow \infty} \int_{\Omega}\left\langle\frac{\nabla_{x} f(x, s, h \xi)}{h}, \varphi(x)\right\rangle \mathrm{d} x \\
& =\lim _{h \rightarrow \infty} \int_{\Omega} \frac{1}{h}\left\langle\nabla_{x} f(x, s, h \xi)-\nabla_{x} f(x, s, 0), \varphi(x)\right\rangle \mathrm{d} x \leq c_{0}\|\varphi\|_{\infty}|\xi|
\end{aligned}
$$

and from this inequality we get at once that $f^{\infty}(\cdot, s, \xi) \in \mathrm{BV}(\Omega)$ and $\left|D_{x} f^{\infty}(\cdot, s, \xi)\right|(\Omega) \leq c_{0}|\xi|$.

Fix $(s, \xi) \in \mathbb{R} \times \mathbb{R}^{N}$. Recalling that $f(\cdot, s, \xi) \in W^{1,1}(\Omega)$, from (5.1) we get that there exists a set $N_{s, \xi}$, with $\mathcal{H}^{N-1}\left(N_{s, \xi}\right)=0$, such that for any $h \in \mathbb{N}$ the function $f(\cdot, s, h \xi)$ is approximately continuous in $\Omega \backslash N_{s, \xi}$. 
Let us fix $x_{0} \in \Omega \backslash N_{s, \xi}$ and $\varepsilon>0$ and let $\delta$ and $L$ be the quantities provided by the assumption (ii). Then for any $\rho \in(0, \delta)$ and $h>L \vee 1$ we get, recalling also (5.4),

$$
f_{B_{\rho}\left(x_{0}\right)}\left|f^{\infty}(x, s, \xi)-f^{\infty}\left(x_{0}, s, \xi\right)\right| \mathrm{d} x \leq \frac{1}{h} f_{B_{\rho}\left(x_{0}\right)}\left|f(x, s, h \xi)-f\left(x_{0}, s, h \xi\right)\right| \mathrm{d} x+2 \varepsilon(1+\Lambda(1+|\xi|)) .
$$

Thus, letting first $\rho \rightarrow 0$ and then $\varepsilon \rightarrow 0$ in the inequality above, we get that $f^{\infty}(\cdot, s, \xi)$ is approximately continuous at $x_{0}$. Hence, the assertion follows.

Lemma 5.5. Assume that $f, f^{\infty}: \Omega \times \mathbb{R} \times \mathbb{R}^{N} \rightarrow \mathbb{R}$ satisfy (5.2)-(5.4) and (5.12). Then, there exists $N_{0} \subset \Omega$ (independent of $(s, \xi)$ ), with $\mathcal{H}^{N-1}\left(N_{0}\right)=0$, such that

$$
\lim _{\varepsilon \rightarrow 0^{+}} f_{B_{\varepsilon}(x)}\left|f^{\infty}(y, s, \xi)-f^{\infty}(x, s, \xi)\right| \mathrm{d} y=0
$$

for every $(s, \xi) \in \mathbb{R} \times \mathbb{R}^{N}$ and every $x \in \Omega \backslash N_{0}$.

Proof. From (5.12), we get that (5.13) holds for every $(s, \xi) \in \mathbb{R} \times \mathbb{R}^{N}$ and every $x \in \Omega \backslash N_{s, \xi}$, with $\mathcal{H}^{N-1}\left(N_{s, \xi}\right)=0$. Now, let $\Sigma_{0}=\left\{\left(s_{k}, \xi_{k}\right)\right\}$ be a countable dense set in $\mathbb{R} \times \mathbb{R}^{N}$ and set $N_{0}=\bigcup_{k} N_{s_{k}, \xi_{k}}$. Clearly, $\mathcal{H}^{N-1}\left(N_{0}\right)=0$ and (5.13) holds for every $x \in \Omega \backslash N_{0}$ and every $\left(s_{k}, \xi_{k}\right) \in \Sigma_{0}$. Therefore, observing that (5.9) implies that $\xi \mapsto f^{\infty}(x, s, \xi)$ is Lipschitz continuous with a constant not depending on $(x, s)$, and recalling (5.10), it follows that (5.13) actually holds for every $(s, \xi) \in \mathbb{R} \times \mathbb{R}^{N}$ and every $x \in \Omega \backslash N_{0}$.

Lemma 5.6. Let $f, f^{\infty}$ satisfy the same assumptions as in Lemma 5.5 and (5.11). Then, for every $\nu \in \mathbb{S}^{N-1}$, there exists $N_{\nu} \subset \pi_{\nu^{\perp}}(\Omega)$ (independent of $s$ ), with $\mathcal{H}^{N-1}\left(N_{\nu}\right)=0$, such that the function $x_{\nu} \mapsto f^{\infty}\left(x_{\nu}^{\perp}, x_{\nu}, s, \nu\right)$ is continuous for every $x_{\nu}^{\perp} \in \pi_{\nu^{\perp}}(\Omega) \backslash N_{\nu}$ and every $s \in \mathbb{R}$.

Proof. By Lemma 4.2, it follows that for every $(s, \nu) \in \mathbb{R} \times \mathbb{S}^{N-1}$ there exists a set $N_{s, \nu} \subset \pi_{\nu^{\perp}}(\Omega)$, with $\mathcal{H}^{N-1}\left(N_{s, \nu}\right)=0$, such that for every $x_{\nu}^{\perp} \in \pi_{\nu^{\perp}}(\Omega) \backslash N_{s, \nu}$ the function $x_{\nu} \mapsto f^{\infty}\left(x_{\nu}^{\perp}, x_{\nu}, s, \nu\right)$ is continuous in $\Omega_{x_{\nu}}$. Now, let $\Sigma_{0}=\left\{s_{k}\right\}$ be a countable dense set in $\mathbb{R}$ and set $N_{\nu}=\bigcup_{k} N_{s_{k}, \nu}$. Clearly, $\mathcal{H}^{N-1}\left(N_{\nu}\right)=0$ and, for every $x_{\nu}^{\perp} \in \pi_{\nu^{\perp}}(\Omega) \backslash N_{\nu}$ and every $s_{k} \in \Sigma_{0}$, the function $x_{\nu} \mapsto f^{\infty}\left(x_{\nu}^{\perp}, x_{\nu}, s_{k}, \nu\right)$ is continuous in $\Omega_{x \frac{\perp}{\nu}}$. By using (5.10) as in the previous proof, it follows that the function $x_{\nu} \mapsto f^{\infty}\left(x_{\nu}^{\perp}, x_{\nu}, s, \nu\right)$ actually is continuous in $\Omega_{x_{\nu}}$, for every $s \in \mathbb{R}$ and every $x_{\nu}^{\perp} \in \pi_{\nu^{\perp}}(\Omega) \backslash N_{\nu}$.

Lemma 5.7. Let $f, f^{\infty}$ be as in Lemma 5.6. Let $D_{0}=\left\{\nu_{j}\right\}$ be a countable sequence of directions in $\mathbb{S}^{N-1}$. There exists a set $\mathcal{G} \subset \Omega$ such that, for every $s \in \mathbb{R}$ and every $\nu_{j} \in D_{0}$, each point $x=\left(x_{\nu_{j}}^{\perp}, x_{\nu_{j}}\right) \in \mathcal{G}$ is a point of approximate continuity for $f^{\infty}\left(\cdot, s, \nu_{j}\right)$ and the function $f^{\infty}\left(\cdot, x_{\nu_{j}}, s, \nu_{j}\right)$ is approximately continuous at $x_{\nu_{j}}^{\perp} \in \Omega_{x_{\nu_{j}}}$. Moreover, $\mathcal{H}^{N-1}(S \backslash \mathcal{G})=0$ for any countably $\mathcal{H}^{N-1}$-rectifiable set $S \subset \Omega$ such that

$$
\mathcal{H}^{N-1}\left(\left\{x \in S: \nu^{S}(x)= \pm \nu_{j}\right\}\right)=0 \quad \text { for all } \nu_{j} \in D_{0}
$$

Proof. For every $\nu_{j} \in D_{0}$ and every $s \in \mathbb{R}$, set

$$
G_{\nu_{j}}^{s}=\left\{x=\left(x_{\nu_{j}}^{\perp}, x_{\nu_{j}}\right) \in \Omega \backslash N_{0}: \lim _{\varepsilon \rightarrow 0^{+}} f_{Q_{\nu_{j}}^{\perp}(x, \varepsilon)}\left|f^{\infty}\left(z_{\nu_{j}}^{\perp}, x_{\nu_{j}}, s, \nu_{j}\right)-f^{\infty}\left(x_{\nu_{j}}^{\perp}, x_{\nu_{j}}, s, \nu_{j}\right)\right| \mathrm{d} z_{\nu_{j}}^{\perp}=0\right\}
$$

where $Q_{\nu_{j}}^{\perp}(x, \varepsilon)=\pi_{\nu_{j}^{\perp}}\left(Q_{\nu_{j}}(x, \varepsilon)\right)$ and $N_{0}$ is the set given by Lemma 5.5. By Remark 4.7, it follows that $\mathcal{H}^{N-1}\left(S \backslash G_{\nu_{j}}^{s}\right)=0$. We consider the set $\mathcal{G}^{s}=\bigcap_{j} G_{\nu_{j}}^{s}$; then, for every $s \in \mathbb{R}, \mathcal{H}^{N-1}\left(S \backslash \mathcal{G}^{s}\right)=0$. Now, let $\left\{s_{k}\right\}$ be a countable dense subset of $\mathbb{R}$ and, for every $k \in \mathbb{N}, \mathcal{G}^{s_{k}}$ be the corresponding set, constructed as above. Finally, set $\mathcal{G}=\bigcap_{k} \mathcal{G}^{s_{k}}$. Clearly, $\mathcal{H}^{N-1}(S \backslash \mathcal{G})=0$. Moreover, as a consequence (5.10) and the density of $\left\{s_{k}\right\}$, 
we have that, for every $\nu_{j} \in D_{0}$ and every $x \in \mathcal{G}$,

$$
\lim _{\varepsilon \rightarrow 0^{+}} f_{Q_{\nu_{j}}^{\perp}(x, \varepsilon)}\left|f^{\infty}\left(z_{\nu_{j}}^{\perp}, x_{\nu_{j}}, s, \nu_{j}\right)-f^{\infty}\left(x_{\nu_{j}}^{\perp}, x_{\nu_{j}}, s, \nu_{j}\right)\right| \mathrm{d} z_{\nu_{j}}^{\perp}=0
$$

for every $s \in \mathbb{R}$.

\section{Main Result}

As we pointed out in the introduction, it has been already proven, for instance in $[5,14,15]$, under suitable regularity assumptions on the integrand function $f$, that the functional defined in (5.6) provides a "natural" extension of the functional (5.5) from $W^{1,1}(\Omega)$ to $\mathrm{BV}(\Omega)$. In the next theorem we state that the same result still holds under the different assumptions on $f$ considered here.

Theorem 6.1. Assume that $f: \Omega \times \mathbb{R} \times \mathbb{R}^{N} \rightarrow \mathbb{R}$ is a Borel function satisfying (5.1)-(5.4). Let $F: \mathrm{BV}(\Omega) \times$ $\mathcal{A}(\Omega) \rightarrow[0,+\infty]$ be the functional defined in $(5.5)$ and $\bar{F}$ be the relaxation of $F$. Assume also that (5.11) and (5.12) hold. Then, $\bar{F}(u, \cdot)$ is the trace of a finite Radon measure on $\mathcal{A}(\Omega)$, and

$$
\bar{F}(u, A)=\int_{A} f(x, u, \nabla u) \mathrm{d} x+\int_{A} f^{\infty}\left(x, \widetilde{u}, \frac{D^{c} u}{\left|D^{c} u\right|}\right) \mathrm{d}\left|D^{c} u\right|+\int_{J_{u} \cap A}\left(\int_{u^{-}(x)}^{u^{+}(x)} f^{\infty}\left(x, s, \nu_{u}\right) \mathrm{d} s\right) \mathrm{d} \mathcal{H}^{N-1}(x)
$$

for every $A \in \mathcal{A}(\Omega)$ and every $u \in \operatorname{BV}(\Omega)$.

We start by observing that under the assumptions of Theorem 6.1 above it is well known that for any $u \in \mathrm{BV}(\Omega)$ the function $\bar{F}(u, \cdot)$ is the trace of a finite Radon measure on $\mathcal{A}(\Omega)$ and that for all $A \in \mathcal{A}(\Omega)$

$$
0 \leq \bar{F}(u, A) \leq c\left(\mathcal{L}^{N}(A)+|D u|(A)\right)
$$

Hence, to prove Theorem 6.1 we have to establish the two inequalities

$$
\begin{array}{ll}
\mathcal{F}(u, A) \leq \bar{F}(u, A) & \text { for all } A \in \mathcal{A}(\Omega) \text { and } u \in \operatorname{BV}(\Omega), \\
\mathcal{F}(u, A) \geq \bar{F}(u, A) & \text { for all } A \in \mathcal{A}(\Omega) \text { and } u \in \operatorname{BV}(\Omega) .
\end{array}
$$

The first one is an immediate consequence of next theorem which, in turn, follows from a more general lower semicontinuity result [7], Theorem 1.1 .

Theorem 6.2. Assume that $f: \Omega \times \mathbb{R} \times \mathbb{R}^{N} \rightarrow[0,+\infty)$ is a locally bounded Borel function, satisfying (5.1)-(5.3). Then, for every $A \in \mathcal{A}(\Omega)$ the functional $\mathcal{F}(\cdot, A): \mathrm{BV}(\Omega) \rightarrow[0,+\infty)$ defined in $(5.6)$ is lower semicontinuous with respect to the $L^{1}$-topology.

Inequality (ii) is established in the next theorem.

Theorem 6.3. Assume that $f: \Omega \times \mathbb{R} \times \mathbb{R}^{N} \rightarrow \mathbb{R}$ is a Borel function satisfying (5.2)-(5.4), (5.11) and (5.12). Then, for every $A \in \mathcal{A}(\Omega)$ and every $u \in \mathrm{BV}(\Omega), \mathcal{F}(u, A) \geq \bar{F}(u, A)$.

Following [15], Proof of Theorem 1.3, we fix $u \in \operatorname{BV}(\Omega)$ and consider the Radon-Nikodým derivatives of $\bar{F}(u, \cdot)$ with respect to the Lebesgue measure $\mathcal{L}^{N}$, to the total variation of the Cantor measure $\left|D^{c} u\right|$ and to the Hausdorff measure $\mathcal{H}^{N-1}\left\lfloor S_{u}\right.$, respectively. In order to obtain Theorem 6.3 , we will prove that

$$
\frac{\mathrm{d} \bar{F}(u, \cdot)}{\mathrm{d} \mathcal{L}^{N}}\left(x_{0}\right) \leq f\left(x_{0}, u\left(x_{0}\right), \nabla u\left(x_{0}\right)\right) \quad \text { for } \mathcal{L}^{N} \text {-almost every } x_{0} \in \Omega,
$$




$$
\frac{\mathrm{d} \bar{F}(u, \cdot)}{\mathrm{d}\left|D^{c} u\right|}\left(x_{0}\right) \leq f^{\infty}\left(x_{0}, \widetilde{u}\left(x_{0}\right), \frac{D^{c} u}{\left|D^{c} u\right|}\left(x_{0}\right)\right) \quad \text { for }\left|D^{c} u\right| \text {-almost every } x_{0} \in \Omega,
$$

$$
\frac{\mathrm{d} \bar{F}(u, \cdot)}{\mathrm{d} \mathcal{H}^{N-1}\left\lfloor J_{u}\right.}\left(x_{0}\right) \leq \int_{u^{-}\left(x_{0}\right)}^{u^{+}\left(x_{0}\right)} f^{\infty}\left(x_{0}, s, \nu_{u}\left(x_{0}\right)\right) \mathrm{d} s \quad \text { for } \mathcal{H}^{N-1} \text {-almost every } x_{0} \in J_{u} .
$$

Inequality (L) is proven in [15] (Th. 1.3, part (i)), under the only assumptions (5.2)-(5.4), hence, we have to prove $(\mathrm{C})$ and $(\mathrm{J})$. To this purpose let us define the following coercive functional associated to $\bar{F}$ by setting

$$
\bar{F}_{1}(u, A):=\bar{F}(u, A)+|D u|(A) .
$$

Proposition 6.4. Assume that $f: \Omega \times \mathbb{R} \times \mathbb{R}^{N} \rightarrow \mathbb{R}$ is a Borel function satisfying (5.2)-(5.4) and (5.12). Then, (C) holds; i.e., for every $u \in \mathrm{BV}(\Omega)$,

$$
\frac{\mathrm{d} \bar{F}(u, \cdot)}{\mathrm{d}\left|D^{c} u\right|}\left(x_{0}\right) \leq f^{\infty}\left(x_{0}, \widetilde{u}\left(x_{0}\right), \frac{D^{c} u}{\left|D^{c} u\right|}\left(x_{0}\right)\right) \quad \text { for }\left|D^{c} u\right| \text {-almost every } x_{0} \in \Omega .
$$

Proof. By Lemma 3.9 of [3] for $\left|D^{c} u\right|$-almost every $x_{0} \in \Omega$, there exists a double indexed sequence $\left\{t_{\varepsilon}^{k}, u_{\varepsilon}^{k}\right\}$ such that, for every $k \in \mathbb{N}$,

$$
t_{\varepsilon}^{k} \rightarrow+\infty, \quad \varepsilon t_{\varepsilon}^{k} \rightarrow 0^{+}, \quad u_{\varepsilon}^{k} \rightarrow \widetilde{u}\left(x_{0}\right) \quad \text { as } \varepsilon \rightarrow 0^{+},
$$

$$
\begin{aligned}
\frac{\mathrm{d} \bar{F}_{1}(u, \cdot)}{\mathrm{d}\left|D^{c} u\right|}\left(x_{0}\right) & =\frac{\mathrm{d} \bar{F}(u, \cdot)}{\mathrm{d}\left|D^{c} u\right|}\left(x_{0}\right)+1 \\
& =\lim _{k \rightarrow+\infty} \limsup _{\varepsilon \rightarrow 0^{+}} \frac{\inf \left\{\bar{F}_{1}\left(v, Q_{\nu}^{k}\left(x_{0}, \varepsilon\right)\right): v \in \operatorname{BV}\left(Q_{\nu}^{k}\left(x_{0}, \varepsilon\right)\right),\left.v\right|_{\partial Q_{\nu}^{k}\left(x_{0}, \varepsilon\right)}=u_{\varepsilon}^{k}+\left\langle t_{\varepsilon}^{k} \nu, x-x_{0}\right\rangle\right\}}{k^{N-1} \varepsilon^{N} t_{\varepsilon}^{k}},
\end{aligned}
$$

where $\nu=\frac{\mathrm{d} D^{c} u}{\mathrm{~d}\left|D^{c} u\right|}\left(x_{0}\right),|\nu|=1$, and $Q_{\nu}^{k}\left(x_{0}, \varepsilon\right):=x_{0}+\varepsilon Q_{\nu}^{k}$, with

$$
Q_{\nu}^{k}:=R_{\nu}\left((-k / 2, k / 2)^{N-1} \times(-1 / 2,1 / 2)\right),
$$

and $R_{\nu}$ denotes a rotation such that $R_{\nu} e_{N}=\nu$. Fix $x_{0} \in \Omega$ so that all the limits above exist and are finite. Moreover, since by Lemma 5.5 there exists $N_{0} \subset \Omega$, with $\mathcal{H}^{N-1}\left(N_{0}\right)=0$ (hence, $\left|D^{c} u\right|\left(N_{0}\right)=0$ ), so that $f^{\infty}(\cdot, s, \xi$ ) is approximately continuous at $x_{0}$ for every $(s, \xi) \in \mathbb{R} \times \mathbb{R}^{N}$, we may assume with no loss of generality that $x_{0} \in \Omega \backslash N_{0}$. Then, taking into account (5.8), (5.4) and (6.1), we have

$$
\begin{aligned}
\frac{\mathrm{d} \bar{F}(u, \cdot)}{\mathrm{d}\left|D^{c} u\right|}\left(x_{0}\right)+1 & \leq \liminf _{k \rightarrow+\infty} \limsup _{\varepsilon \rightarrow 0^{+}} \frac{1}{k^{N-1} \varepsilon^{N} t_{\varepsilon}^{k}} \bar{F}_{1}\left(u_{\varepsilon}^{k}+\left\langle t_{\varepsilon}^{k} \nu, x-x_{0}\right\rangle, Q_{\nu}^{k}\left(x_{0}, \varepsilon\right)\right) \\
& \leq \liminf _{k \rightarrow+\infty} \limsup _{\varepsilon \rightarrow 0^{+}} f_{Q_{\nu}^{k}\left(x_{0}, \varepsilon\right)} \frac{f\left(x, u_{\varepsilon}^{k}+\left\langle t_{\varepsilon}^{k} \nu, x-x_{0}\right\rangle, t_{\varepsilon}^{k} \nu\right)}{t_{\varepsilon}^{k}} \mathrm{~d} x+1 \\
& \leq \liminf _{k \rightarrow+\infty} \limsup _{\varepsilon \rightarrow 0^{+}} f_{Q_{\nu}^{k}\left(x_{0}, \varepsilon\right)}\left(f^{\infty}\left(x, u_{\varepsilon}^{k}+\left\langle t_{\varepsilon}^{k} \nu, x-x_{0}\right\rangle, \nu\right)+\frac{f\left(x, u_{\varepsilon}^{k}+\left\langle t_{\varepsilon}^{k} \nu, x-x_{0}\right\rangle, 0\right)}{t_{\varepsilon}^{k}}\right) \mathrm{d} x+1 \\
& \leq \liminf _{k \rightarrow+\infty} \limsup _{\varepsilon \rightarrow 0^{+}} f_{Q_{\nu}^{k}\left(x_{0}, \varepsilon\right)} f^{\infty}\left(x, u_{\varepsilon}^{k}+\left\langle t_{\varepsilon}^{k} \nu, x-x_{0}\right\rangle, \nu\right) \mathrm{d} x+1,
\end{aligned}
$$

which implies

$$
\frac{\mathrm{d} \bar{F}(u, \cdot)}{\mathrm{d}\left|D^{c} u\right|}\left(x_{0}\right) \leq \liminf _{k \rightarrow+\infty} \limsup _{\varepsilon \rightarrow 0^{+}} f_{Q_{\nu}^{k}\left(x_{0}, \varepsilon\right)} f^{\infty}\left(x, u_{\varepsilon}^{k}+\left\langle t_{\varepsilon}^{k} \nu, x-x_{0}\right\rangle, \nu\right) \mathrm{d} x .
$$


Hence, in order to conclude, it is enough to prove that for all $k \in \mathbb{N}$

$$
\limsup _{\varepsilon \rightarrow 0^{+}} f_{Q_{\nu}^{k}\left(x_{0}, \varepsilon\right)} f^{\infty}\left(x, u_{\varepsilon}^{k}+\left\langle t_{\varepsilon}^{k} \nu, x-x_{0}\right\rangle, \nu\right) \mathrm{d} x \leq f^{\infty}\left(x_{0}, \widetilde{u}\left(x_{0}\right), \nu\right) .
$$

By (5.10), the approximate continuity of the function $f^{\infty}\left(\cdot, \widetilde{u}\left(x_{0}\right), \nu\right)$ in $x_{0} \in \Omega$ and (6.1), it follows that for every $k \in \mathbb{N}$

$$
\begin{aligned}
& \limsup _{\varepsilon \rightarrow 0^{+}} f_{Q_{\nu}^{k}\left(x_{0}, \varepsilon\right)} f^{\infty}\left(x, u_{\varepsilon}^{k}+\left\langle t_{\varepsilon}^{k} \nu, x-x_{0}\right\rangle, \nu\right) \mathrm{d} x \\
& \quad \leq \limsup _{\varepsilon \rightarrow 0^{+}} f_{Q_{\nu}^{k}\left(x_{0}, \varepsilon\right)} f^{\infty}\left(x, \widetilde{u}\left(x_{0}\right), \nu\right) \mathrm{d} x+\underset{\varepsilon \rightarrow 0^{+}}{\limsup } f_{Q_{\nu}^{k}\left(x_{0}, \varepsilon\right)} \Lambda\left|u_{\varepsilon}^{k}+\left\langle t_{\varepsilon}^{k} \nu, x-x_{0}\right\rangle-\widetilde{u}\left(x_{0}\right)\right| \mathrm{d} x \\
& \quad \leq f^{\infty}\left(x_{0}, \widetilde{u}\left(x_{0}\right), \nu\right)+\Lambda \limsup _{\varepsilon \rightarrow 0^{+}}\left[\left|u_{\varepsilon}^{k}-\widetilde{u}\left(x_{0}\right)\right|+f_{Q_{\nu}^{k}\left(x_{0}, \varepsilon\right)} t_{\varepsilon}^{k}\left|x-x_{0}\right| \mathrm{d} x\right] \\
& \leq f^{\infty}\left(x_{0}, \widetilde{u}\left(x_{0}\right), \nu\right)+\Lambda \limsup _{\varepsilon \rightarrow 0^{+}}\left[\left|u_{\varepsilon}^{k}-\widetilde{u}\left(x_{0}\right)\right|+\varepsilon t_{\varepsilon}^{k} \sqrt{(N-1) k^{2}+1}\right]=f^{\infty}\left(x_{0}, \widetilde{u}\left(x_{0}\right), \nu\right) .
\end{aligned}
$$

Thus (6.3) is proved. Hence, the assertion follows.

Proposition 6.5. Let $f$ satisfy the assumptions of Theorem 6.3. Then,

$$
\frac{\mathrm{d} \bar{F}(u, \cdot)}{\mathrm{d} \mathcal{H}^{N-1}\left\lfloor J_{u}\right.}\left(x_{0}\right) \leq \int_{u^{-}\left(x_{0}\right)}^{u^{+}\left(x_{0}\right)} f^{\infty}\left(x_{0}, s, \nu_{u}\left(x_{0}\right)\right) \mathrm{d} s
$$

for every $u \in \mathrm{BV}(\Omega)$ and for $\mathcal{H}^{N-1}$-almost every $x_{0} \in J_{u}$.

Proof. Let $u \in \mathrm{BV}(\Omega)$. We will prove that for $\mathcal{H}^{N-1}$-a.e. $x_{0} \in J_{u}$

$$
\frac{\mathrm{d} \bar{F}(u, \cdot)}{\mathrm{d} \mathcal{H}^{N-1}\left\lfloor J_{u}\right.}\left(x_{0}\right)=\lim _{r \rightarrow 0^{+}} \frac{\bar{F}\left(u, B_{r}\left(x_{0}\right)\right)}{\mathcal{H}^{N-1}\left\lfloor J_{u}\left(B_{r}\left(x_{0}\right)\right)\right.} \leq \int_{u^{-}\left(x_{0}\right)}^{u^{+}\left(x_{0}\right)} f^{\infty}\left(x_{0}, s, \nu_{u}\left(x_{0}\right)\right) \mathrm{d} s .
$$

By Remark 3.1 the set $\Phi_{u}$, defined by

$$
\Phi_{u}=\left\{\nu \in \mathbb{S}^{N-1}: \mathcal{H}^{N-1}\left(\left\{x \in J_{u}: \nu_{u}(x)= \pm \nu\right\}\right)>0\right\}
$$

is at most countable. Given a countable dense subset $D_{0}$ of directions in $\mathbb{S}^{N-1} \backslash \Phi_{u}$, we apply Lemma 5.7, with $S=J_{u}$. Thus, there exists a set $\mathcal{G} \subset \Omega$, such that, for every $s \in \mathbb{R}$ and every $\nu \in D_{0}$, if $x=\left(x_{\nu}^{\perp}, x_{\nu}\right) \in \mathcal{G}$, then $f^{\infty}(\cdot, s, \nu)$ is approximately continuous at $x, f^{\infty}\left(\cdot, x_{\nu}, s, \nu\right)$ is approximately continuous at $x_{\nu}^{\perp}$ and $\mathcal{H}^{N-1}\left(J_{u} \backslash \mathcal{G}\right)=0$.

By Theorem 3.7 of [3], for $\mathcal{H}^{N-1}$-almost every $x_{0} \in J_{u} \cap \mathcal{G}$ we have

$$
\begin{aligned}
\frac{\mathrm{d} \bar{F}_{1}(u, \cdot)}{\mathrm{d} \mathcal{H}^{N-1}\left\lfloor J_{u}\right.}\left(x_{0}\right) & =\frac{\mathrm{d} \bar{F}(u, \cdot)}{\mathrm{d} \mathcal{H}^{N-1}\left\lfloor J_{u}\right.}\left(x_{0}\right)+\left|u^{+}\left(x_{0}\right)-u^{-}\left(x_{0}\right)\right| \\
& =\limsup _{\varepsilon \rightarrow 0^{+}} \frac{\inf \left\{\bar{F}_{1}\left(v, Q_{\nu}\left(x_{0}, \varepsilon\right)\right): v \in \operatorname{BV}\left(Q_{\nu}\left(x_{0}, \varepsilon\right)\right),\left.v\right|_{\partial Q_{\nu}\left(x_{0}, \varepsilon\right)}=w_{\nu}\right\}}{\varepsilon^{N-1}},
\end{aligned}
$$

where $\nu=\nu_{u}\left(x_{0}\right), Q_{\nu}\left(x_{0}, \varepsilon\right)=x_{0}+\varepsilon Q_{\nu}, Q_{\nu}$ is defined as in (6.2) with $k=1$, and $w_{\nu}$ is the jump function which takes the value $u^{+}\left(x_{0}\right)$ if $\left\langle x-x_{0}, \nu\right\rangle>0$ and $u^{-}\left(x_{0}\right)$ if $\left\langle x-x_{0}, \nu\right\rangle \leq 0$. Let $\left\{\nu_{j}\right\}$ be a sequence of directions contained in $D_{0}$ converging to $\nu$. Let us fix $\delta>0$; then $Q_{\nu}(0,1-\delta) \subset Q_{\nu_{j}} \subset Q_{\nu}(0,1+\delta)$ for every $j$ sufficiently large.

Let $\phi \in \mathcal{C}_{0}^{\infty}\left(Q_{\nu}(0 ; 1+\delta)\right)$ be a cut-off function such that $\phi(x)=1$ in $Q_{\nu}(0 ; 1-2 \delta), \phi(x)=0$ on $Q_{\nu}(0 ; 1+\delta) \backslash$ $Q_{\nu}(0 ; 1-\delta)$ and $|\nabla \phi| \leq c / \delta$. For every $\varepsilon>0$, set $\phi_{\varepsilon}(x)=\phi\left(\frac{x-x_{0}}{\varepsilon}\right)$, so that $\left|\nabla \phi_{\varepsilon}\right| \leq c / \varepsilon \delta$ and, for every $j \in \mathbb{N}$ 
sufficiently large, set $w_{\varepsilon, \nu, \nu_{j}}(x)=\phi_{\varepsilon}(x) w_{\nu_{j}}(x)+\left(1-\phi_{\varepsilon}(x)\right) w_{\nu}(x)$, where $w_{\nu_{j}}$ is defined as $w_{\nu}$, with $\nu$ replaced by $\nu_{j}$. We note that $w_{\varepsilon, \nu, \nu_{j}}$ satisfies the boundary condition $\left.w_{\varepsilon, \nu, \nu_{j}}\right|_{\partial Q_{\nu}\left(x_{0}, \varepsilon\right)}=w_{\nu}$, so that, by (6.5), we obtain

$$
\frac{\mathrm{d} \bar{F}_{1}(u, \cdot)}{\mathrm{d} \mathcal{H}^{N-1}\left\lfloor J_{u}\right.}\left(x_{0}\right) \leq \limsup _{\varepsilon \rightarrow 0^{+}}\left[\frac{\bar{F}\left(w_{\varepsilon, \nu, \nu_{j}}, Q_{\nu}\left(x_{0}, \varepsilon\right)\right)}{\varepsilon^{N-1}}+\frac{\left|D w_{\varepsilon, \nu, \nu_{j}}\right|\left(Q_{\nu}\left(x_{0}, \varepsilon\right)\right)}{\varepsilon^{N-1}}\right]
$$

Clearly, for every $\varepsilon>0$ and $j \in \mathbb{N}$ sufficiently large,

$$
\begin{aligned}
& \frac{\left|D w_{\varepsilon, \nu, \nu_{j}}\right|\left(Q_{\nu}\left(x_{0}, \varepsilon\right)\right)}{\varepsilon^{N-1}} \\
& \leq \frac{1}{\varepsilon^{N-1}}\left[\int_{Q_{\nu}\left(x_{0}, \varepsilon\right)}\left|\nabla \phi_{\varepsilon}\right|\left|w_{\nu_{j}}-w_{\nu}\right| \mathrm{d} x+\left|D w_{\nu_{j}}\right|\left(Q_{\nu}\left(x_{0}, \varepsilon\right)\right)+\left|D w_{\nu}\right|\left(Q_{\nu}\left(x_{0}, \varepsilon\right) \backslash Q_{\nu}\left(x_{0},(1-2 \delta) \varepsilon\right)\right)\right] \\
& \leq \frac{c}{\varepsilon^{N} \delta} \int_{Q_{\nu}\left(x_{0}, \varepsilon\right)}\left|w_{\nu_{j}}-w_{\nu}\right| \mathrm{d} x+\frac{\left|u^{+}\left(x_{0}\right)-u^{-}\left(x_{0}\right)\right|}{\left|\left\langle\nu, \nu_{j}\right\rangle\right|}+c \delta \leq \frac{c}{\delta} \sin \left(\widehat{\nu \nu_{j}}\right)+\frac{\left|u^{+}\left(x_{0}\right)-u^{-}\left(x_{0}\right)\right|}{\left|\left\langle\nu, \nu_{j}\right\rangle\right|}+c \delta .
\end{aligned}
$$

Moreover, let us approximate the jump function $w_{\varepsilon, \nu, \nu_{j}}$ by means of a sequence of $W^{1,1}$-functions, given by $u_{\varepsilon, \nu, \nu_{j}}^{n}(x)=\phi_{\varepsilon}(x) u_{\varepsilon, \nu_{j}}^{n}(x)+\left(1-\phi_{\varepsilon}(x)\right) u_{\varepsilon, \nu}^{n}(x)$, where

$$
u_{\varepsilon, \nu}^{n}(x):= \begin{cases}u^{+}\left(x_{0}\right) & \text { if } \varepsilon / 2 n \leq\left\langle x-x_{0}, \nu\right\rangle, \\ \left(u^{+}\left(x_{0}\right)-u^{-}\left(x_{0}\right)\right) \frac{n}{\varepsilon}\left\langle x-x_{0}, \nu\right\rangle+\frac{u^{+}\left(x_{0}\right)+u^{-}\left(x_{0}\right)}{2} & \text { if }-\varepsilon / 2 n \leq\left\langle x-x_{0}, \nu\right\rangle \leq \varepsilon / 2 n \\ u^{-}\left(x_{0}\right) & \text { if }\left\langle x-x_{0}, \nu\right\rangle \leq-\varepsilon / 2 n,\end{cases}
$$

and $u_{\varepsilon, \nu_{j}}^{n}$ is defined similarly by replacing $\nu$ with $\nu_{j}$. Clearly, $\left\|u_{\varepsilon, \nu, \nu_{j}}^{n}-w_{\varepsilon, \nu, \nu_{j}}\right\|_{L^{1}\left(Q_{\nu}\left(x_{0}, \varepsilon\right)\right)} \rightarrow 0$ as $n \rightarrow+\infty$, hence, using the lower semicontinuity of $\bar{F},(5.4),(5.8)$, it follows

$$
\begin{aligned}
& \frac{\bar{F}\left(w_{\varepsilon, \nu, \nu_{j}}, Q_{\nu}\left(x_{0}, \varepsilon\right)\right)}{\varepsilon^{N-1}} \leq \liminf _{n \rightarrow+\infty} \frac{1}{\varepsilon^{N-1}} \int_{Q_{\nu}\left(x_{0}, \varepsilon\right)} f\left(x, u_{\varepsilon, \nu, \nu_{j}}^{n}, \nabla u_{\varepsilon, \nu, \nu_{j}}^{n}\right) \mathrm{d} x \\
& \leq \liminf _{n \rightarrow+\infty} \frac{1}{\varepsilon^{N-1}}\left[\int_{Q_{\nu}\left(x_{0}, \varepsilon(1-2 \delta)\right)} f\left(x, u_{\varepsilon, \nu_{j}}^{n}, \nabla u_{\varepsilon, \nu_{j}}^{n}\right) \mathrm{d} x+c \int_{Q_{\nu}\left(x_{0}, \varepsilon\right)}\left[\left|\nabla \phi_{\varepsilon}\right|\left|u_{\varepsilon, \nu}^{n}-u_{\varepsilon, \nu_{j}}^{n}\right|+1\right] \mathrm{d} x\right. \\
& \left.+c \int_{Q_{\nu}\left(x_{0}, \varepsilon\right) \backslash Q_{\nu}\left(x_{0},(1-2 \delta) \varepsilon\right)}\left(\phi_{\varepsilon}\left|\nabla u_{\varepsilon, \nu_{j}}^{n}\right|+\left(1-\phi_{\varepsilon}\right)\left|\nabla u_{\varepsilon, \nu}^{n}\right|+1\right) \mathrm{d} x\right] \\
& \leq \liminf _{n \rightarrow+\infty} \int_{Q_{\nu_{j}}\left(x_{0}, \varepsilon\right)} \varepsilon f\left(x, u_{\varepsilon, \nu_{j}}^{n}, \nabla u_{\varepsilon, \nu_{j}}^{n}\right) \mathrm{d} x+c \varepsilon+\frac{c}{\delta} \sin \left(\widehat{\nu \nu_{j}}\right)+c \delta \\
& \leq \liminf _{n \rightarrow+\infty} \int_{Q_{\nu_{j}}\left(x_{0}, \varepsilon\right)}\left[f^{\infty}\left(x, u_{\varepsilon, \nu_{j}}^{n}, \varepsilon \nabla u_{\varepsilon, \nu_{j}}^{n}\right)+\varepsilon f\left(x, u_{\varepsilon, \nu_{j}}^{n}, 0\right)\right] \mathrm{d} x+c \varepsilon+\frac{c}{\delta} \sin \left(\widehat{\nu \nu_{j}}\right)+c \delta \\
& \leq \liminf _{n \rightarrow+\infty} \int_{Q_{\nu_{j}}\left(x_{0}, \varepsilon\right)} f^{\infty}\left(x, u_{\varepsilon, \nu_{j}}^{n}, \varepsilon \nabla u_{\varepsilon, \nu_{j}}^{n}\right) \mathrm{d} x+c \varepsilon+\frac{c}{\delta} \sin \left(\widehat{\nu \nu_{j}}\right)+c \delta \\
& \leq\left(u^{+}\left(x_{0}\right)-u^{-}\left(x_{0}\right)\right) \liminf _{n \rightarrow+\infty} \int_{Q_{\nu_{j}}^{\perp}\left(x_{0}, \varepsilon\right)}\left[\frac{n}{\varepsilon} \int_{\left(x_{0}\right)_{\nu_{j}}-\varepsilon / 2 n}^{\left(x_{0}\right)_{\nu_{j}}+\varepsilon / 2 n} f^{\infty}\left(x_{\nu_{j}}^{\perp}, x_{\nu_{j}}, u_{\varepsilon, \nu_{j}}^{n}(x), \nu_{j}\right) \mathrm{d} x_{\nu_{j}}\right] \mathrm{d} x_{\nu_{j}}^{\perp} \\
& +c \varepsilon+\frac{c}{\delta} \sin \left(\widehat{\nu \nu_{j}}\right)+c \delta,
\end{aligned}
$$


where $Q_{\nu_{j}}^{\perp}\left(x_{0}, \varepsilon\right)=\pi_{\nu_{j}^{\perp}}\left(Q_{\nu_{j}}\left(x_{0}, \varepsilon\right)\right)$. We note that the function $u_{\varepsilon, \nu_{j}}^{n}$ actually depends only on $x_{\nu_{j}}$, so that, by the change of variable $s=u_{\varepsilon, \nu_{j}}^{n}\left(x_{\nu_{j}}\right)$, we obtain

$$
x_{\nu_{j}}=\rho_{n, \varepsilon}(s)=\frac{\varepsilon}{n\left(u^{+}\left(x_{0}\right)-u^{-}\left(x_{0}\right)\right)}\left(s-\frac{u^{+}\left(x_{0}\right)+u^{-}\left(x_{0}\right)}{2}\right)+\left(x_{0}\right)_{\nu_{j}}
$$

and $\rho_{n, \varepsilon}(s) \rightarrow\left(x_{0}\right)_{\nu_{j}}$, when $n \rightarrow+\infty$; so that, by dominated convergence theorem and Lemma 5.6 , we have

$$
\begin{aligned}
\left(u^{+}\left(x_{0}\right)-\right. & \left.u^{-}\left(x_{0}\right)\right) \limsup _{\varepsilon \rightarrow 0^{+}} \liminf _{n \rightarrow+\infty} f_{Q_{\nu_{j}}^{\perp}\left(x_{0}, \varepsilon\right)}\left[\frac{n}{\varepsilon} \int_{\left(x_{0}\right)_{\nu_{j}}-\varepsilon / 2 n}^{\left(x_{0}\right)_{\nu_{j}}+\varepsilon / 2 n} f^{\infty}\left(x_{\nu_{j}}^{\perp}, x_{\nu_{j}}, u_{\varepsilon, \nu_{j}}^{n}\left(x_{\nu_{j}}\right), \nu_{j}\right) \mathrm{d} x_{\nu_{j}}\right] \mathrm{d} x_{\nu_{j}}^{\perp} \\
& =\limsup _{\varepsilon \rightarrow 0^{+}} \liminf _{n \rightarrow+\infty} f_{Q_{\nu_{j}}^{\perp}\left(x_{0}, \varepsilon\right)}\left[\int_{u^{-}\left(x_{0}\right)}^{u^{+}\left(x_{0}\right)} f^{\infty}\left(x_{\nu_{j}}^{\perp}, \rho_{n, \varepsilon}(s), s, \nu_{j}\right) \mathrm{d} s\right] \mathrm{d} x_{\nu_{j}}^{\perp} \\
& \leq \limsup _{\varepsilon \rightarrow 0^{+}} f_{Q_{\nu_{j}}^{\perp}\left(x_{0}, \varepsilon\right)}\left[\int_{u^{-}\left(x_{0}\right)}^{u^{+}\left(x_{0}\right)} \lim _{n \rightarrow+\infty} f^{\infty}\left(x_{\nu_{j}}^{\perp}, \rho_{n, \varepsilon}(s), s, \nu_{j}\right) \mathrm{d} s\right] \mathrm{d} x_{\nu_{j}}^{\perp} \\
& \leq \limsup _{\varepsilon \rightarrow 0^{+}} f_{Q_{\nu_{j}}^{\perp} u\left(x_{0}, \varepsilon\right)}\left[\int_{u^{-}\left(x_{0}\right)}^{u^{+}\left(x_{0}\right)} f^{\infty}\left(x_{\nu_{j}}^{\perp},\left(x_{0}\right)_{\nu_{j}}, s, \nu_{j}\right) \mathrm{d} s\right] \mathrm{d} x_{\nu_{j}}^{\perp} \\
& =\int_{u^{-}\left(x_{0}\right)}^{u^{+}\left(x_{0}\right)}\left[\lim _{\varepsilon \rightarrow 0^{+}} f_{Q_{\nu_{j}}^{\perp}\left(x_{0}, \varepsilon\right)} f^{\infty}\left(x_{\nu_{j}}^{\perp},\left(x_{0}\right)_{\nu_{j}}, s, \nu_{j}\right) \mathrm{d} x_{\nu_{j}}^{\perp}\right] \mathrm{d} s=\int_{u^{-}\left(x_{0}\right)}^{u^{+}\left(x_{0}\right)} f^{\infty}\left(x_{0}, s, \nu_{j}\right) \mathrm{d} s
\end{aligned}
$$

where the last equality is due to the approximate continuity at $\left(x_{0}\right)_{\nu_{j}}^{\perp}$ of $f^{\infty}\left(\cdot,\left(x_{0}\right)_{\nu_{j}}, s, \nu_{j}\right)$, for every $s \in \mathbb{R}$. By (6.5)-(6.9), we obtain, letting $\varepsilon \rightarrow 0^{+}$,

$$
\frac{\mathrm{d} \bar{F}(u, \cdot)}{\mathrm{d} \mathcal{H}^{N-1}\left\lfloor J_{u}\right.}\left(x_{0}\right)+\left|u^{+}\left(x_{0}\right)-u^{-}\left(x_{0}\right)\right| \leq \int_{u^{-}\left(x_{0}\right)}^{u^{+}\left(x_{0}\right)} f^{\infty}\left(x_{0}, s, \nu_{j}\right) \mathrm{d} s+\frac{c}{\delta} \sin \left(\widehat{\nu \nu_{j}}\right)+c \delta+\frac{\left|u^{+}\left(x_{0}\right)-u^{-}\left(x_{0}\right)\right|}{\left|\left\langle\nu, \nu_{j}\right\rangle\right|}
$$

Now, taking into account the Lipschitz continuity of $f^{\infty}$ with respect to the last variable and letting first $j \rightarrow+\infty$ and then $\delta \rightarrow 0^{+}$we get

$$
\frac{\mathrm{d} \bar{F}(u, \cdot)}{\mathrm{d} \mathcal{H}^{N-1}\left\lfloor J_{u}\right.}\left(x_{0}\right)+\left|u^{+}\left(x_{0}\right)-u^{-}\left(x_{0}\right)\right| \leq \int_{u^{-}\left(x_{0}\right)}^{u^{+}\left(x_{0}\right)} f^{\infty}\left(x_{0}, s, \nu_{u}\left(x_{0}\right)\right) \mathrm{d} s+\left|u^{+}\left(x_{0}\right)-u^{-}\left(x_{0}\right)\right| .
$$

Hence, the assertion follows.

We are now in position to give the proof of Theorem 6.1.

Proof of Theorem 6.1. Taking into account Theorem 6.2, [15] (Th. 1.3, part (i)), and Propositions 6.4 and 6.5, we obtain the assertion for any function $u \in \mathrm{BV}(\Omega)$ and any $A \in \mathcal{A}(\Omega)$.

\section{REFERENCES}

[1] M. Amar and V. De Cicco, Relaxation in BV for a class of functionals without continuity assumptions. NoDEA (to appear).

[2] L. Ambrosio, N. Fusco and D. Pallara, Functions of bounded variation and free discontinuity problems. Oxford University Press, New York (2000).

[3] G. Bouchitté, I. Fonseca and L. Mascarenhas, A global method for relaxation. Arch. Rat. Mech. Anal. 145 (1998) 51-98.

[4] G. Buttazzo, Semicontinuity, Relaxation and Integral Representation Problems in the Calculus of Variations. Pitman Res. Notes Math., Longman, Harlow (1989).

[5] G. Dal Maso, Integral representation on $B V(\Omega)$ of $\Gamma$-limits of variational integrals. Manuscripta Math. 30 (1980) $387-416$.

[6] G. Dal Maso, An Introduction to $\Gamma$-convergence. Birkhäuser, Boston (1993).

[7] V. De Cicco, N. Fusco and A. Verde, On $L^{1}$-lower semicontinuity in $B V(\Omega)$. J. Convex Analysis 12 (2005) 173-185. 
[8] V. De Cicco, N. Fusco and A. Verde, A chain rule formula in $B V(\Omega)$ and its applications to lower semicontinuity. Calc. Var. Partial Differ. Equ. 28 (2007) 427-447.

[9] V. De Cicco and G. Leoni, A chain rule in $L^{1}(\operatorname{div} ; \Omega)$ and its applications to lower semicontinuity. Calc. Var. Partial Differ. Equ. 19 (2004) 23-51.

[10] E. De Giorgi and T. Franzoni, Su un tipo di convergenza variazionale. Atti Accad. Naz. Lincei Rend. Cl. Sci. Fis. Mat. Natur. 58 (1975) 842-850.

[11] E. De Giorgi and T. Franzoni, Su un tipo di convergenza variazionale. Rend. Sem. Mat. Brescia 3 (1979) 63-101.

[12] L.C. Evans and R.F. Gariepy, Lecture Notes on Measure Theory and Fine Properties of Functions. CRC Press, Boca Raton (1992).

[13] H. Federer, Geometric measure theory. Springer-Verlag, Berlin (1969).

[14] I. Fonseca and G. Leoni, Some remarks on lower semicontinuity. Indiana Univ. Math. J. 49 (2000) 617-635.

[15] I. Fonseca and G. Leoni, On lower semicontinuity and relaxation. Proc. R. Soc. Edinb. Sect. A Math. 131 (2001) 519-565.

[16] I. Fonseca and S. Müller, Quasi-convex integrands and lower semicontinuity in L $L^{1}$. SIAM J. Math. Anal. 23 (1992) $1081-1098$.

[17] I. Fonseca and S. Müller, Relaxation of quasiconvex functionals in $\operatorname{BV}\left(\Omega, \mathbb{R}^{p}\right)$ for integrands $f(x, u, \nabla u)$. Arch. Rat. Mech. Anal. 123 (1993) 1-49.

[18] N. Fusco, M. Gori and F. Maggi, A remark on Serrin's Theorem. NoDEA 13 (2006) 425-433.

[19] E. Giusti, Minimal Surfaces and Functions of Bounded Variation. Birkhäuser, Boston (1984).

[20] M. Gori and P. Marcellini, An extension of the Serrin's lower semicontinuity theorem. J. Convex Anal. 9 (2002) $475-502$.

[21] M. Gori, F. Maggi and P. Marcellini, On some sharp conditions for lower semicontinuity in L $L^{1}$. Diff. Int. Eq. 16 (2003) 51-76.

[22] A.I. Vol'pert and S.I. Hudjaev, Analysis in Classes of Discontinuous Functions and Equations of Mathematical Physics. Martinus \& Nijhoff Publishers, Dordrecht (1985). 